\title{
Improvements in deterministic error modeling and calibration of inertial sensors and magnetometers
}

\author{
Gorkem Secer, Billur Barshan* \\ Department of Electrical and Electronics Engineering, Bilkent University, Bilkent, TR-06800 Ankara, Turkey
}

\section{A R T I C L E I N F O}

\section{Article history:}

Received 29 January 2016

Received in revised form 14 June 2016

Accepted 20 June 2016

Available online 1 July 2016

\section{Keywords:}

Inertial sensors

Accelerometer

Gyroscope

Magnetometer

Deterministic error modeling

Measurement model

In-field calibration

Model parameter estimation

Ellipsoid parameter estimation

Levenberg-Marquardt algorithm

Particle swarm optimization

\begin{abstract}
A B S T R A C T
We consider the deterministic modeling, calibration, and model parameter estimation of two commonly employed inertial measurement units based on real test data acquired from a flight motion simulator. Each unit comprises three tri-axial devices: an accelerometer, a gyroscope, and a magnetometer. We perform the deterministic error modeling and calibration of accelerometers based on an improved measurement model, and the technique we propose for gyroscopes lowers costs by eliminating the need for additional sensors and relaxing the test bed requirement. We present an extended measurement model for magnetometers that reduces calibration errors by modeling orientation-dependent hard-iron errors in a gimbaled angular position-control machine. While we employ the model-based LevenbergMarquardt optimization algorithm for the parameter estimation of accelerometers and magnetometers, we use a model-free evolutionary optimization algorithm (particle swarm optimization) for estimating the calibration parameters of gyroscopes. Errors are considerably reduced as a result of proper modeling and calibration.
\end{abstract}

(c) 2016 Elsevier B.V. All rights reserved.

\section{Introduction}

Inertial sensors were mainly only used in aeronautics and maritime applications until the nineties because of the high cost associated with the high-accuracy requirements. With developments in micro-electro-mechanical systems (MEMS), the availability of small, lower-cost, medium-performance inertial sensors has opened up new possibilities for their use, such as the recognition of daily activities [1], physical therapy and home-based rehabilitation [2], biomechanics [3], detecting and classifying falls $[4,5]$, shock and vibration analysis, navigation of unmanned vehicles [6-8], and state estimation and dynamic modeling of legged robots [9].

Inertial measurement units (IMUs) typically contain gyroscopes and accelerometers, sometimes used in conjunction with magnetometers. Each device can be sensitive around a single axis or multiple axes (usually two or three). An accelerometer detects specific force, which is proportionate to the acceleration of the sensor

\footnotetext{
* Corresponding author.

E-mail addresses: gorkem.secer@ceng.metu.edu.tr (G. Secer), billur@ee.bilkent.edu.tr (B. Barshan).
}

relative to an inertial reference frame along its axis of sensitivity. A gyroscope senses the angular rate about an axis of sensitivity with respect to an inertial reference frame $[10,11]$. Magnetometers measure the magnetic field strength at a given location superposed with the Earth's magnetic field [12]. They are used in a wide range of disciplines, from archaeology [13] to vehicle navigation and control [14].

Consumer-grade inertial sensors have attracted much interest recently because of their decreasing cost due to developments in MEMS technology [15]. Measurements by inertial sensors often deviate from the ground truth since the devices suffer from various error types, which can be constant or time varying. The rate output of accelerometers and gyroscopes needs to be integrated twice or once to obtain the linear or angular position, respectively. Because of the integration process, even very small errors at the output accumulate very rapidly and the position error becomes considerably large in a few seconds and starts drifting in time (i.e., proportionate with the time cube for the linear and the time square for the angular position) [16]. This effect is exacerbated for low-grade sensors. Consumer-grade inertial sensors can be used for longer periods of time on their own if modeled and calibrated properly, but may need to be corrected from time to time by an external aid that provides an absolute reference for the ground truth $[17,18]$. Thus, to improve 


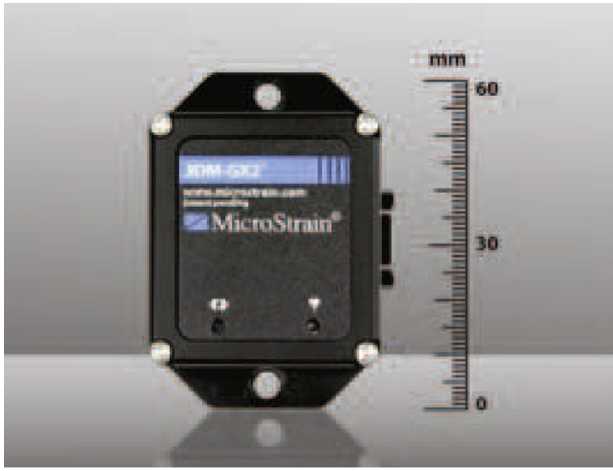

(a)

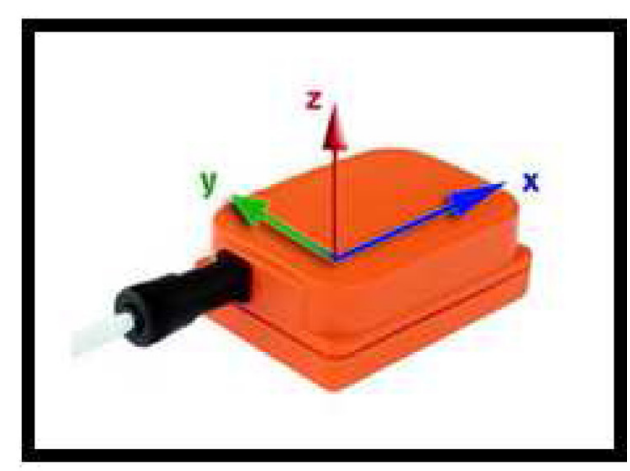

(b)

Fig. 1. The two sensor units used in this study: (a) MicroStrain 3DM-GX2 [22] and (b) Xsens MTx [23].

the accuracy of linear and angular position estimates, it is necessary to characterize and model the errors at the sensor output precisely. The same holds for magnetometers that suffer from various error types.

Most previous works have divided the calibration problem into two distinct parts (deterministic and stochastic modeling) because of their different mathematical natures $[10,19,20]$. Here, we follow the same approach and focus on deterministic calibration only. Stochastic calibration is considered in a different study [21].

Working from their raw outputs, we consider the deterministic calibration of two widely used consumer-grade IMUs and compare their performances: MicroStrain's 3DM-GX2 [22] and Xsens' MTx [23], depicted in Fig. 1, with their technical specifications provided in the respective references. The units are small, light, and comprised of three tri-axial devices: an accelerometer, a gyroscope, and a magnetometer. The main objective of this study is to effectively model and estimate the units' deterministic calibration parameters so that both their stand-alone and aided performances can be improved.

Motivated by insights gained from earlier work, we propose improved models and algorithmic ideas and implement them to improve the sensor calibration process. The main contributions of this article are threefold:

- We propose an improvement to the traditional measurement model used in $1 g$ tests for modeling the deterministic errors of accelerometers. The method's effectiveness is shown through experiments, and the results are compared with those of the traditional model.

- We employ a low-cost calibration technique to estimate the error components associated with gyroscopes. Our technique is based on comparing the attitude of the IMU, calculated by integrating the gyroscope measurements, with the reference attitude provided by a 3-DoF flight motion simulator (FMS). In this way, we eliminate the need for any additional sensors to perform the calibration, unlike previously used low-cost gyroscope calibration techniques. Another novel aspect of this work is that to estimate the model parameters that minimize the attitude error of gyroscopes, we use a global optimization algorithm [particle swarm optimization (PSO)] instead of gradient-based techniques, to avoid convergence to local minima.

- We propose an extended sensor measurement model for magnetometers that reduces calibration errors by modeling the orientation-dependent magnetic disturbances in gimbaled angular position-control machines. We experimentally verify that incorporating in the model the relative motion between the magnetometer and the magnetic distortion sources in the environment enhances the calibration accuracy.
The rest of this article is organized as follows: In Section 2, we first develop individual deterministic sensor measurement models for each type of sensor and then propose a unified measurement model for all three sensor types. Section 3 describes the data acquisition experiments conducted for calibrating the sensors and briefly reviews geometric and algebraic parameter estimation techniques. We then present our model parameter estimation results based on the acquired data and propose an extended measurement model for magnetometers. We compare the two units in terms of measurement quality based on the results of deterministic calibration. In Section 4, we summarize our contributions, make concluding remarks, and provide some directions for future research. In the appendices, we provide background information on the two optimization algorithms that we use for parameter estimation.

\section{Sensor measurement models}

The general measurement model of the sensors evaluated in this study is given by:

$\vec{e}_{m}=\vec{h}(\vec{e}, \vec{\theta})+\vec{n}$

where $\vec{h}(\vec{e}, \vec{\theta}): \mathbb{R}^{3} \times \mathbb{R}^{\operatorname{dim}(\vec{\theta})} \rightarrow \mathbb{R}^{3}$. The $\vec{e}_{m}, \vec{e}$, and $\vec{n} \in \mathbb{R}^{3}$ denote the measured sensor output, the true value of the excitation signal, and the additive stochastic measurement noise vector, respectively. The calibration parameter vector $\vec{\theta}$ involved in this model needs to be estimated accurately in the scope of deterministic calibration.

The following notation is used throughout: The measured sensor output $\vec{e}_{m}$ can be one of $\vec{a}_{m}, \vec{\omega}_{m}$, or $\vec{B}_{m}$, for the accelerometer, gyroscope, and magnetometer, respectively. The true excitation signal $\vec{e}$ can be one of $\vec{a}, \vec{\omega}$, or $\vec{B}$, which represent the true values of the specific acceleration, angular rate, and magnetic field strength vectors. A vector $\vec{u}$ expressed with respect to a coordinate frame $f$ is denoted by $\vec{u}^{f}$, and the rotational transformation matrix $\mathbf{C}_{f_{1}}^{f_{2}}$, transforms a vector $\vec{u}^{f_{1}}$ from frame $f_{1}$ to $f_{2}$ as $\vec{u}^{f_{2}}=\mathbf{C}_{f_{1}}^{f_{2}} \vec{u}^{f_{1}}$. Orthonormal unit vectors of the $x, y$, and $z$ axes of a given frame $f$ are respectively denoted by $\vec{i}^{f}, \vec{j}^{f}$, and $\vec{k}^{f}$.

To develop the deterministic measurement model of the sensors, we first need to introduce several coordinate frames:

- the north-east-down (NED) frame is shown in Fig. 2, with unit vectors $\vec{i}^{\mathrm{NED}}, \vec{j}^{\mathrm{NED}}$, and $\vec{k}^{\mathrm{NED}}$, which point to the north, east, and down directions of the Earth, respectively.

- the platform base frame $(p)$ is an orthogonal frame fixed to the base of the rotating platform onto which the sensor units are mounted, and does not move with the platform.

- the sensor enclosure frame $(q)$ corresponds to the orthogonal axes of the sensor's mechanical casing. Due to manufacturing tolerances and packaging issues, in practice, this frame cannot 


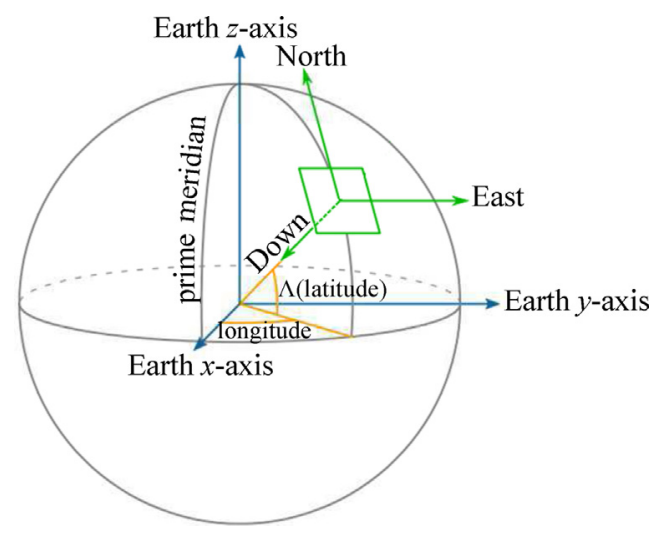

Fig. 2. The NED frame and the Earth's frame of reference. Adopted from [67].

be perfectly aligned with the sensor's actual sensitivity axes ( $s$ frame). This frame moves with the platform onto which the sensor units are attached.

- the orthogonal sensor sensitivity frame $(r)$ is the idealized, orthogonal sensor sensitivity frame.

- the non-orthogonal sensor sensitivity frame $(s)$ represents the set of the sensor's actual sensitivity axes. Deviation from orthogonality stems from manufacturing tolerances in general, and may affect navigation performance significantly.

As shown in Fig. 3(a), for the platform that we use in the calibration tests, the $\vec{k}$ unit vectors of the NED and $p$ frames are coincident and their respective $\vec{i}$ and $\vec{j}$ unit vectors lie on the horizontal plane, making an angle $\beta$ with each other. This is because the base of the facility where experiments are conducted was leveled with the North-East plane. Thus, the relationship between the two frames can be described by a rotational transformation $\mathbf{C}_{\mathrm{NED}}^{p}$ about the $\vec{k}$ axis by $\beta$ :

$\mathbf{C}_{\mathrm{NED}}^{p}=\left[\begin{array}{ccc}\cos \beta & \sin \beta & 0 \\ -\sin \beta & \cos \beta & 0 \\ 0 & 0 & 1\end{array}\right]$

The matrix $\mathbf{C}_{p}^{q}$ can be expressed mathematically by a sequence of basic rotations as $\mathbf{C}_{p}^{q}=\mathbf{R}_{x}(\phi) \mathbf{R}_{y}(\gamma) \mathbf{R}_{z}(\psi)$, where $\phi, \gamma$, and $\psi$ are the angles of rotation about the $x, y$, and $z$ axes of the $p$ frame, respectively. The basic rotation matrices are given by:

$\mathbf{R}_{x}(\phi)=\left[\begin{array}{ccc}1 & 0 & 0 \\ 0 & \cos \phi & \sin \phi \\ 0 & -\sin \phi & \cos \phi\end{array}\right]$

$\mathbf{R}_{y}(\gamma)=\left[\begin{array}{ccc}\cos \gamma & 0 & -\sin \gamma \\ 0 & 1 & 0 \\ \sin \gamma & 0 & \cos \gamma\end{array}\right]$

$\mathbf{R}_{z}(\psi)=\left[\begin{array}{ccc}\cos \psi & \sin \psi & 0 \\ -\sin \psi & \cos \psi & 0 \\ 0 & 0 & 1\end{array}\right]$

Similarly, the package misalignment matrix $\mathbf{C}_{q}^{r}$ represents the misalignment between frames $q$ and $r$ as $\mathbf{C}_{q}^{r}=\mathbf{R}_{x}\left(\epsilon_{x}\right) \mathbf{R}_{y}\left(\epsilon_{y}\right) \mathbf{R}_{z}\left(\epsilon_{z}\right)$, where $\epsilon_{x}, \epsilon_{y}$, and $\epsilon_{z}$ are the mounting misalignment angles about the $x, y$, and $z$ axes of the $q$ frame, respectively.

The unit vectors of the $r$ and $s$ frames are such that $\vec{i}^{r}$ and $\vec{i}^{s}$ are coincident, $\vec{j}^{r}$ lies along the remaining perpendicular component of $\vec{j}$ after its projection onto $\vec{i}^{r}, \vec{k}^{r}$ points in the same direction as the component of $\vec{k}^{s}$, perpendicular to the plane spanned by $\vec{i}^{r}$ and $\vec{j}^{r}$, as illustrated in Fig. 4. This kinematic relation between frames $r$ and $s$ can be modeled by the non-orthogonalization (cross-axis sensitivity) matrix:

$\mathbf{T}_{r}^{s}=\left[\begin{array}{ccc}1 & 0 & 0 \\ \sin \left(\alpha_{1}\right) & \cos \left(\alpha_{1}\right) & 0 \\ \cos \left(\alpha_{3}\right) & \sin \left(\alpha_{2}\right) \sin \left(\alpha_{3}\right) & \cos \left(\alpha_{2}\right) \sin \left(\alpha_{3}\right)\end{array}\right]$

where $\alpha_{i}, i \in\{1,2,3\}$ are the sensor-to-sensor axis misalignment angles (Fig. 4). With this definition, a vector $\vec{u}^{r}$ can be transformed from frame $r$ to $s$ as $\vec{u}^{s}=\mathbf{T}_{r}^{s} \vec{u}^{r}$.

According to a first-order approximation at the sensor output [19], we introduce a diagonal scale factor error matrix $\mathbf{S}$ such that $\operatorname{diag}(\mathbf{S})=\left[\begin{array}{lll}S_{x} & S_{y} & S_{z}\end{array}\right]$. A factor of $(\mathbf{I}+\mathbf{S})$ multiplies the true excitation signal in the measurement model, where $\mathbf{I}$ is the $3 \times 3$ identity matrix, so that the output of each sensor axis is scaled by a different amount in general.

When the input to the sensor is zero, the deviation of the output from the zero level is the bias error [6,19], denoted by $\vec{b}=\left[\begin{array}{lll}b_{x} & b_{y} & b_{z}\end{array}\right]^{T}$ in this study. The bias error may change with the sensor's operating temperature and may drift in time. Since the experiments are conducted over short periods of time in this study, we assume a constant bias level.

The deterministic error components and the transformation matrices introduced above are common to both inertial sensors and magnetometers.

\subsection{Accelerometer measurement model}

After incorporating the error components described in the previous section and the related transformations into the sensor measurement model in Eq. (1), we obtain the traditional first-order measurement model of accelerometers [24-27]:

$\vec{a}_{m}=(\mathbf{I}+\mathbf{S}) \mathbf{T}_{r}^{s} \mathbf{C}_{q}^{r} \mathbf{C}_{p}^{q} \vec{a}^{p}+\vec{b}+\vec{n}$

Here, $\vec{a}_{m}$ is the acceleration measured along the sensitivity axes of the accelerometer (the $s$ frame), whereas the reference for the true value of the excitation signal $\vec{a}^{p}$ is the $p$ frame. We note that the composite matrix multiplying $\vec{a}^{p}$ above represents a transformation from the $p$ to the $s$ frame and corrects for the scale factor error.

We propose the following improved measurement model:

$\vec{a}_{m}=(\mathbf{I}+\mathbf{S}) \mathbf{T}_{r}^{S} \mathbf{C}_{q}^{r} \mathbf{C}_{p}^{q} \mathbf{C}_{\mathrm{NED}}^{p} \vec{a}^{\mathrm{NED}}+\vec{b}+\vec{n}$

The main difference between the two models is that in the proposed model, the matrix $\mathbf{C}_{\mathrm{NED}}^{p}$ is included so that the reference for the true acceleration $\vec{a}^{\mathrm{NED}}$ is now the NED frame [28]. Accordingly, the $\vec{h}(\vec{e}, \vec{\theta})$ for accelerometers is $(\mathbf{I}+\mathbf{S}) \mathbf{T}_{r}^{s} \mathbf{C}_{q}^{r} \mathbf{C}_{p}^{q} \mathbf{C}_{\mathrm{NED}}^{p} \vec{a}^{\mathrm{NED}}+\vec{b}$. The composite matrix multiplying $\vec{a}^{\text {NED }}$ here represents a transformation from the NED to the $s$ frame and corrects for the scale factor error as well.

\subsection{Gyroscope measurement model}

The first-order measurement model of gyroscopes is given by:

$\vec{\omega}_{m}=(\mathbf{I}+\mathbf{S}) \mathbf{T}_{r}^{s} \mathbf{C}_{q}^{r} \mathbf{C}_{p}^{q} \vec{\omega}^{p}+\vec{b}+\vec{n}$

The $\vec{\omega}_{m}$ is measured along the sensor's sensitivity axes (the $s$ frame) but the reference for the true excitation signal is the $p$ frame. The composite matrix multiplying $\vec{\omega}^{p}$ above corresponds to a transformation from the $p$ to the $s$ frame and corrects for the scale factor error. 


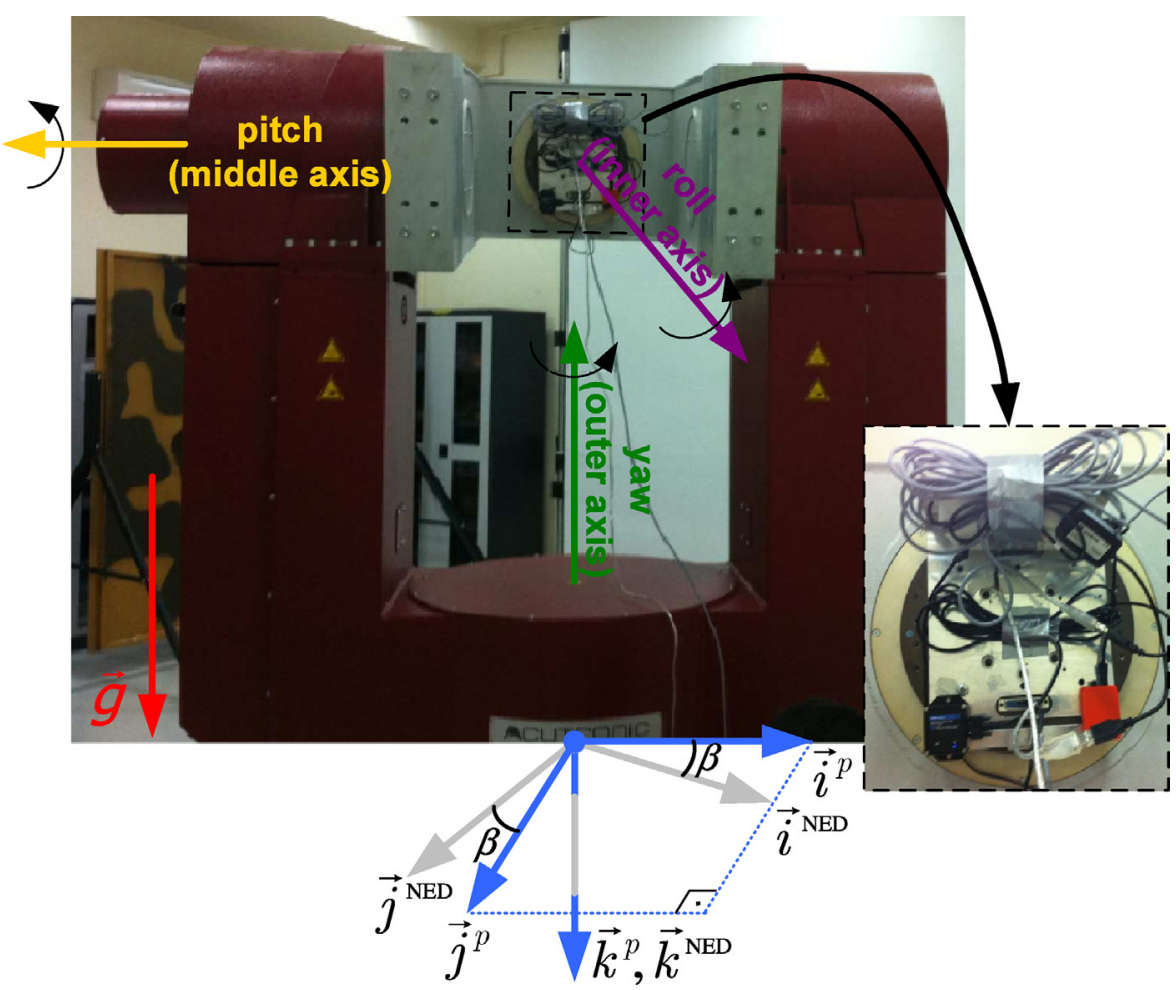

(a) Step 1

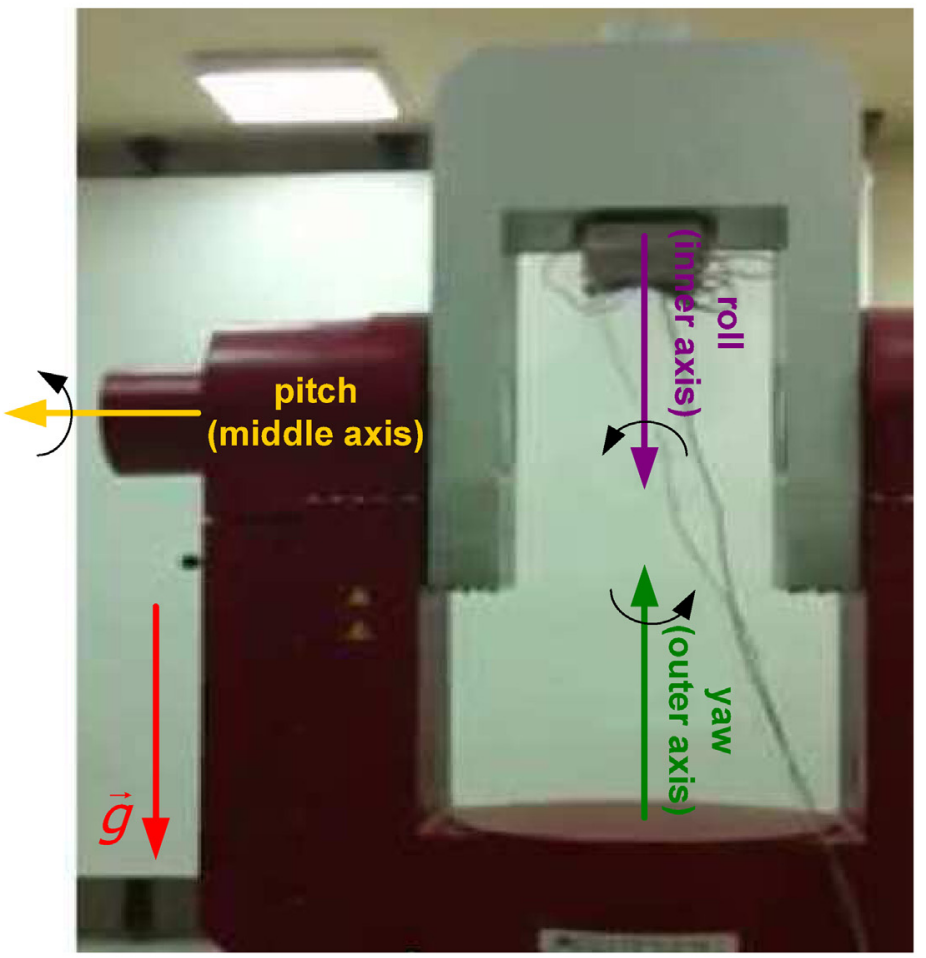

(b) Step 3

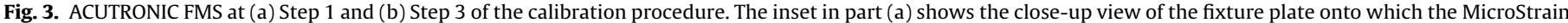
and Xsens units are attached.

\subsection{Magnetometer measurement model}

Magnetometers are used to estimate the attitude of frame $q$ with respect to the NED frame by measuring the Earth's magnetic field vector, denoted by $\vec{B}^{\mathrm{NED}}$, with respect to the NED frame. However, in the real world, especially in indoor environments, magnetometers are exposed to more than just $\vec{B}^{\mathrm{NED}}$. The presence of ferromagnetic materials and/or sources that radiate magnetic fields in the vicinity of the sensor are the main factors that affect the magnetic field vector $\vec{B}_{m}$ measured by the sensor and cause deterioration. 


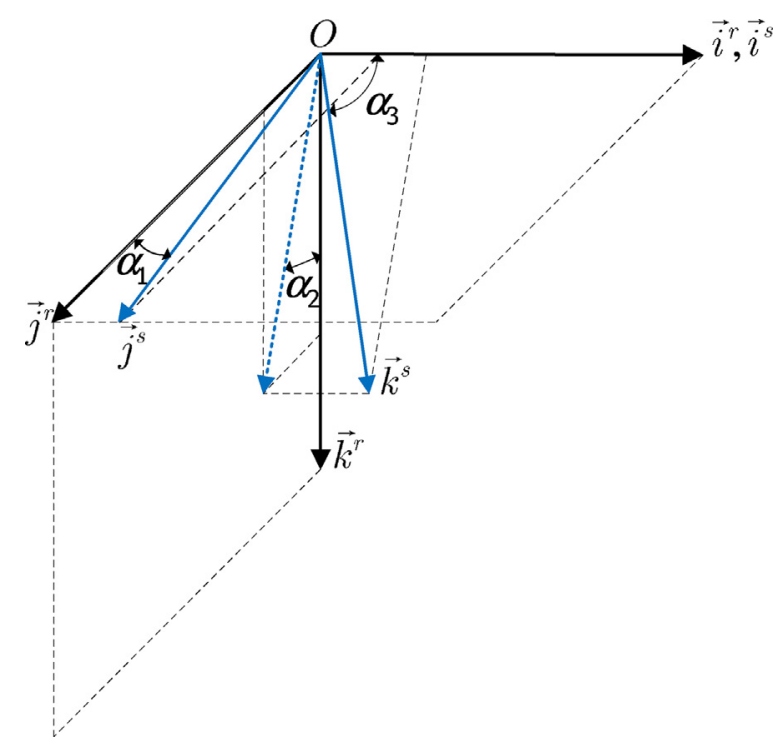

Fig. 4. The $r$ and $s$ frames and their unit vectors.

The related errors are divided into two classes: soft- and hard-iron errors [29,30]:

Soft-iron errors are caused by the interaction of an external magnetic field with ferromagnetic materials in the vicinity of the sensor [29]. The magnetic permeability of materials has a direct influence on this interaction. Soft-iron errors can be represented by a symmetric matrix $\mathbf{K}$. Since its elements satisfy $k_{i j}=k_{j i}$, where $i, j=1,2,3$ because of symmetry, the matrix has six independent elements.

Hard-iron errors are time-invariant, undesired magnetic fields generated by ferromagnetic materials with permanent magnetism that are part of the structure of the platform onto which the sensors are mounted, or are part of equipment installed near the magnetometer [30]. Magnetic fields radiated by actuators such as electrical motors on gimbaled systems and constant magnetic fields in the test environment are some examples. The effect of these on the sensor axes could be time varying and orientation dependent because of the relative motion between the sensors and the environment. The resultant magnetic field is a combination of $\vec{B}^{\mathrm{NED}}$ and $\delta \vec{B}$, where $\delta \vec{B}=\left[\begin{array}{lll}\delta B_{x} & \delta B_{y} & \delta B_{z}\end{array}\right]^{T}$.

Modifying Eq. (5) to include $\mathbf{K}$ and $\delta \vec{B}$ for soft- and hard-iron errors and replacing the excitation signal and the measured output with $\vec{B}^{\mathrm{NED}}$ and $\vec{B}_{m}$, respectively, we obtain the traditional first-order measurement model of magnetometers [29,31]:

$\vec{B}_{m}=(\mathbf{I}+\mathbf{S}) \mathbf{T}_{r}^{s} \mathbf{C}_{q}^{r}\left(\mathbf{K} \mathbf{C}_{p}^{q} \mathbf{C}_{\mathrm{NED}}^{p} \vec{B}^{\mathrm{NED}}+\delta \vec{B}\right)+\vec{b}+\vec{n}$

It follows that for magnetometers, $\vec{h}(\vec{e}, \vec{\theta})=(\mathbf{I}+\mathbf{S})$ $\mathbf{T}_{r}^{s} \mathbf{C}_{q}^{r}\left(\mathbf{K C}_{p}^{q} \mathbf{C}_{\mathrm{NED}}^{p} \vec{B}^{\mathrm{NED}}+\delta \vec{B}\right)+\vec{b}$. Here, the excitation signal $\vec{B}^{\mathrm{NED}}$ is with respect to the NED frame, whereas $\vec{B}_{m}$ is measured along the sensitivity axes of the magnetometer (the $s$ frame). Thus, the composite matrix multiplying $\vec{B}^{\mathrm{NED}}$ in the above equation represents a transformation from the NED to the $s$ frame and corrects for the scale factor and soft-iron errors.

\subsection{The unified measurement model}

The measurement models of inertial sensors and magnetometers, represented by Eqs. (5)-(7) have some similarity. Generalizing the three equations into a unified measurement model:

$\vec{e}_{m}=\mathbf{H} \vec{e}+\vec{b}^{\prime}+\vec{n}$
From Eq. (5), the coefficient matrix $\mathbf{H} \triangleq(\mathbf{I}+\mathbf{S}) \mathbf{T}_{r}^{s} \mathbf{C}_{q}^{r} \mathbf{C}_{p}^{q} \mathbf{C}_{\mathrm{NED}}^{p}$ for accelerometers, whereas, based on Eqs. (6) and (7), respectively, $\mathbf{H} \triangleq(\mathbf{I}+\mathbf{S}) \mathbf{T}_{r}^{s} \mathbf{C}_{q}^{r} \mathbf{C}_{p}^{q}$ for gyroscopes and $\mathbf{H} \triangleq(\mathbf{I}+\mathbf{S}) \mathbf{T}_{r}^{s} \mathbf{C}_{q}^{r} \mathbf{K} \mathbf{C}_{p}^{q} \mathbf{C}_{\mathrm{NED}}^{p}$ for magnetometers. Here, $\vec{b}^{\prime}$ is the unified bias vector, which is simply equal to $\vec{B}$ for inertial sensors, whereas $\vec{b}^{\prime}=(\mathbf{I}+\mathbf{S}) \mathbf{T}_{r}^{s} \mathbf{C}_{q}^{r} \delta \vec{B}+\vec{b}$ for magnetometers.

Having defined $\mathbf{H}$ and $\vec{b}^{\prime}$, we note that both are functions of the calibration parameter vector $\vec{\theta}$. There are no additional constraints on the choice of $\mathbf{H}$ and $\vec{b}$. Furthermore, since sensors might undergo dynamic motions during the experiments, the term $\mathbf{C}_{p}^{q}$, appearing in $\mathbf{H}$, is a time-dependent matrix, as is the excitation signal $\vec{e}$ (whose time-dependence we have not yet shown explicitly to keep the notation simple). Thus, $\mathbf{H}(\vec{\theta}, t): \mathbb{R}^{\operatorname{dim}(\vec{\theta})} \times \mathbb{R} \rightarrow \mathbb{R}^{3} \times \mathbb{R}^{3}$ and $\overrightarrow{b^{\prime}}(\vec{\theta}): \mathbb{R}^{\operatorname{dim}(\vec{\theta})} \rightarrow \mathbb{R}^{3}$, where we assume a constant bias model. The vector $\vec{\theta}$ is determined by the parameters involved in the given measurement models and will be described for each different sensor type in the next section, while $t$ denotes the time instant corresponding to the measurement. Finally, for all three types of sensors considered, we state that:

$\vec{e}_{m}(t)=\vec{h}(\vec{e}, \vec{\theta}, t)+\vec{n}(t)=\mathbf{H}(\vec{\theta}, t) \vec{e}(t)+\vec{b}^{\prime}(\vec{\theta})+\vec{n}(t)$

\section{Sensor calibration}

Calibration is a multi-step procedure for improving position estimates by inverting the unified measurement model given in Eq. (9) to estimate the calibration parameter vector $\vec{\theta}$. The first step requires designing an experimental procedure for data acquisition. In the second step, we implement parameter estimation algorithms based on the data acquired during the first step.

\subsection{Data acquisition tests}

Various test procedures are available to acquire data for estimating the calibration parameters of the different sensor types. Deterministic calibration methods can be divided into two classes: traditional and in-field.

Traditional methods are usually employed in the aerospace industry to calibrate tactical-grade IMUs, requiring highly expensive and specialized machines with accurate angular positionand/or velocity-control capability. Reference inputs are applied to the sensors at multiple positions to compare their measurements with and estimate the calibration parameters. The precise attitude at each position is required and reference inputs are used in component-wise form for this purpose. The precision of such machinery directly affects the estimation accuracy of the model parameters and the related cost increases proportionately. The choice of the test procedure depends on the type of machinery used.

With the accelerated development of low-cost inertial sensors, the need for low-cost in-field calibration techniques emerged, where the sensors are calibrated while operating in the field, without the need for any additional actuators or sensors. Because of the limitations of these techniques, however, attitude information and consequently, component-wise reference inputs, such as the components of $\vec{g}^{\mathrm{NED}}$ and $\vec{B}^{\mathrm{NED}}$, are not available to calibrate accelerometers and magnetometers, respectively. Instead, the magnitudes (norms) of the reference inputs are compared with those of the sensor measurement vectors since $\left\|\vec{g}^{\mathrm{NED}}\right\|=\left\|\mathbf{C}_{p}^{q} \mathbf{C}_{\mathrm{NED}}^{p} \vec{g}^{\mathrm{NED}}\right\|$, thus, eliminating the need for projecting excitation signals onto the $q$ frame [24,32]. However, when low-cost in-field calibration techniques are used, it is neither possible to detect the misalignment matrix $\mathbf{C}_{q}^{r}$ nor $\mathbf{C}_{\mathrm{NED}}^{p}$. Furthermore, when using only the magnitudes of the reference inputs, the choice of the form of the matrix $\mathbf{T}_{r}^{s}$ is no longer trivial. This is because the order of the orthogonalization 
process determines the unknown matrix $\mathbf{C}_{q}^{r}$, and affects the results, as the installation is still made with respect to the IMU casing. This is not the case when using component-wise reference inputs, where the matrix $\mathbf{C}_{q}^{r}$ compensates for the difference.

Below, we give a brief description of the typical calibration approach for each sensor type used in this study:

- Accelerometer: Accelerometers are traditionally calibrated by using angular position-control or centrifuge machines. In the former, the accelerometer is positioned and held stationary at various known reference orientations throughout the test. This is known as the multi-position or the $1 g$ test. Calibration parameters are estimated based on the acquired sensor measurements and the reference accelerations associated with the reference orientations (i.e., the local gravity vector $\vec{g}$ ) [10]. The limitation of this procedure is that the reference acceleration inputs applied to the sensors are restricted to the $[-g,+g]$ interval, which may result in inaccurate calibration outside this interval. When centrifuge machines are used, reference acceleration inputs are not necessarily restricted to the $[-g,+g]$ interval and higher acceleration values are sustainable [33]. Deterministic error components can then be identified in the same way as in the former procedure.

- Gyroscope: The way calibration tests are performed for gyroscopes depends on the quality grade of the device. High-precision gyroscopes are capable of measuring the Earth's angular velocity, enabling the use of multi-position tests. Gyroscopes can be positioned at reference orientations and the calibration parameters can be estimated by comparing the sensor measurements with the reference angular rate (the Earth's angular velocity $\vec{\omega}$ ) at these positions [10]. However, lower-grade MEMS gyroscopes cannot be calibrated this way because they are not capable of sensing $\vec{\omega}$ [34]; they need to be exposed to different reference angular velocities that can be provided by an angular position-control machine or a single-axis rate table [35]. The latter allows accurate calibration over a broader operation range compared to the former. The calibration parameters can be estimated by comparing the sensor measurements with the reference angular rates [36-38]. If such reference angular rates are not available, alternative techniques are required: (i) calibrated accelerometers and/or magnetometers, embedded in the sensor unit together with the gyroscope, can be used to provide the reference information. If accelerometers are employed, measurements at stationary positions are compared with the gravity projected onto the sensor sensitivity axes by an angular transformation computed using gyroscopic measurements [39,40]. However, accelerometers are only able to detect the two angles between the sensor and the local horizontal direction [41]; their measurements cannot resolve the rotation about the local vertical direction (the yaw angle). Alternatively, embedded magnetometers that project the Earth's magnetic field onto the sensor's sensitivity axes as reference information can be used [42]. Calibration is commonly performed by simply rotating the sensors by hand on a flat surface. (ii) when there is no additional sensor embedded in the sensor unit, the gyroscope can be fixed to one of the contact surfaces of a right-angled plate and rotated by hand on a flat surface. In this case, the attitude computed by gyroscope measurements is compared with the reference attitude associated with the plate's configuration [43].

- Magnetometer: Magnetometers are also calibrated based on data acquired during multi-position tests. The reference input is the Earth's local magnetic field vector $\vec{B}^{\text {NED }}$ [29,31,44-46]. Magnetometers need to undergo testing on the platform on which they will be eventually used (e.g., an aircraft or unmanned ground vehicle), and their calibration parameters need to be estimated specifically for this platform. The orientation of the platform is often controllable and it is capable of producing the motions required for the multi-position test $[31,47,48]$. When this is the case, the interior effects of the platform (e.g., the magnetic permeability of the material of the test bed) can be modeled as constant time-invariant distortion since there is no relative motion between the platform and the magnetometer. However, the platform and the magnetometer are usually not isolated from the environment. External magnetic sources such as electric motors or transformers may affect the magnetometer measurements $[29,46]$ and contribute additional time-varying distortion components $[49,50]$. Despite that, in most of the earlier studies, such external distortion components are assumed to be constant for a given calibration platform. Considering this deficiency of the traditional methods, we present an extended measurement model accounting for the time-varying magnetic distortions that are often encountered in non-isolated test platforms, where there is relative motion between the magnetometer and the distortion sources in the environment.

In the present study, we use ACUTRONIC's high-precision 3-DoF FMS to conduct multi-position tests for the deterministic calibration of our sensors. The FMS and its rotation axes are illustrated in Fig. 3 and the technical specifications can be found in [51].

Both units are mounted side by side to the FMS's fixture plate, located on the shaft of the inner axis. This is illustrated in the inset of Fig. 3(a). Then, a trajectory for the FMS axes, which is called a calibration procedure, is determined for the experiments and programmed into the FMS controller computer. The steps of the calibration procedure are summarized below, where the rotation angles are stated according to the right-hand rule so that positive-valued angles indicate a counter-clockwise rotation about the corresponding axis:

1. The inner axis of the FMS is aligned with the ground level, as shown in Fig. 3(a).

2. The inner axis of the FMS is rotated by $270^{\circ}$ in 12 steps of $22.5^{\circ}$ each. The FMS is held stationary at each one of those steps for $12.5 \mathrm{~s}$.

3. The inner axis of the FMS is aligned with the vertical gravity vector $\vec{g}$, as shown in Fig. 3(b).

4. The FMS makes a half turn $\left(180^{\circ}\right)$ around its middle axis while it stops and waits for $12.5 \mathrm{~s}$ at each step of $22.5^{\circ}$.

5. The FMS is taken back to its angular position at Step 3.

6 . The inner axis of the FMS is rotated by $90^{\circ}$.

7. The FMS performs the same motion as in Step 4.

While designing this multi-position calibration procedure, it is necessary to ensure that the accelerometers and magnetometers experience a complete set of reference inputs. This is illustrated in Fig. 5 where the component-wise reference acceleration input applied to the MicroStrain unit is provided as an example. During the calibration procedure, the accelerometer, gyroscope, and magnetometer outputs of both units are recorded simultaneously at a uniform sampling rate of $100 \mathrm{~Hz}$.

To avoid additional disturbance on the measurements that might occur while the FMS is in motion, we only consider the accelerometer and magnetometer measurements acquired during the 12.5 -s periods when the FMS is stationary. The local gravity and magnetic field vectors, $\vec{g}^{\text {NED }}$ and $\vec{B}^{\text {NED }}$, can be calculated/looked up and used as reference inputs in their component-wise form for the accelerometer/magnetometer measurements at these stationary positions, respectively. Thus, the measurements acquired at these stationary positions are kept and processed, while the rest are discarded. The time indices in this subset of $N$ elements are renumbered as a consecutive array. The final form of this subset is denoted by $N_{s}$. 
Component-wise reference input $\vec{a}^{q}$ applied to MicroStrain

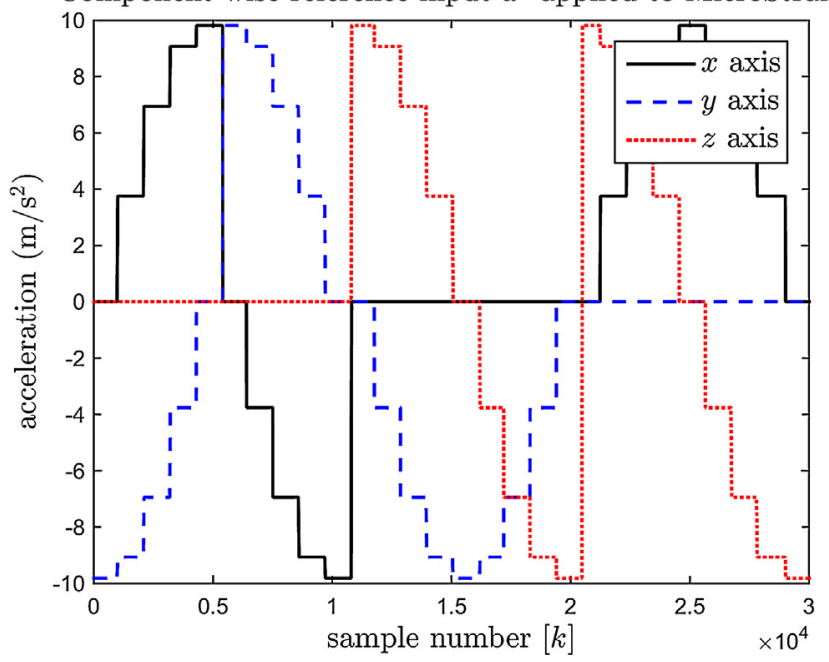

Fig. 5. Component-wise reference input applied to the accelerometer of the MicroStrain unit in its $q$ frame according to the calibration procedure.

Our low-cost consumer-grade gyroscopes cannot be calibrated using the same approach because of the lack of component-wise reference inputs. These devices are not sensitive enough to detect the Earth's angular velocity as a reference input and the true angular rates of the FMS axes are not available. Therefore, there is no reference input or ground truth for the angular rate to directly compare the gyroscope rate outputs with. The FMS is position-controlled and only provides information on the true angular positions, represented by the transformation matrix $\mathbf{C}_{p}^{q}$ between the $p$ and $q$ frames. The $\mathbf{C}_{p}^{q}$ matrices, available when the FMS is stationary, can be used as ground truth for the angular position and compared with the integrated gyroscope rate measurements. Therefore, we keep all gyroscope measurements acquired during the calibration procedure to be able to estimate $\mathbf{C}_{p}^{q}$ when the FMS is stationary.

\subsection{Measurement model parameter estimation}

Another challenge in deterministic calibration is selecting suitable and robust parameter estimation techniques among a variety. The choice depends on the complexity of the sensor model to a great extent. Thus, when orthogonality and misalignment errors (and soft- and hard-iron errors of magnetometers) can be neglected, fundamental linear methods such as batch least-squares are adopted so that measurement equations reduce to a linear system of equations $[41,48,52,53]$. In [54], rank constraints of the linear system of equations are exploited for parameter estimation. However, to adequately compensate for measurement errors, it is essential to consider the nonlinearities in the sensor measurement model. In this regard, ellipsoid parameter estimation techniques are used quite extensively $[29,44,55]$. These techniques are divided into two classes: geometric and algebraic fit methods [56], and are based on different aspects of the calibration models.

We use an ellipsoid parameter estimation approach in this study described by the unified measurement model in Eq. (9). The discrete form of this model is given by:

$\vec{e}_{m}[k]=\vec{h}[\vec{e}, \vec{\theta}, k]+\vec{n}[k]=\mathbf{H}[\vec{\theta}, k] \vec{e}[k]+\vec{b}^{\prime}[\vec{\theta}]+\vec{n}[k]$

Geometric and algebraic parameter estimation techniques estimate the calibration parameters by minimizing the following respective expressions, both of which can be obtained from the discretized model in Eq. (10):

$\underset{\vec{\theta}}{\operatorname{argmin}} \sum_{k}\left\|\vec{e}_{m}[k]-\mathbf{H}[\vec{\theta}, k] \vec{e}[k]-\vec{b}^{\prime}[\vec{\theta}]\right\|$ and

$$
\begin{aligned}
& \underset{\vec{\theta}}{\arg \min _{k}} \sum_{k}\left[\left(\vec{e}_{m}[k]-\vec{b}^{\prime}[\vec{\theta}]\right)^{T}\left(\mathbf{H}^{-1}[\vec{\theta}, k]\right)^{T}\left(\mathbf{H}^{-1}[\vec{\theta}, k]\right)\right. \\
& \left.\left(\vec{e}_{m}[k]-\vec{b}^{\prime}[\vec{\theta}]\right)-\vec{e}^{T}[k] \vec{e}[k]\right]^{2}
\end{aligned}
$$

It is shown in [56] that geometric techniques are superior to algebraic techniques in terms of fitness accuracy, as expected since Eq. (11) contains more extensive information than Eq. (12). However, the exact knowledge of the excitation signal $\vec{e}[k]$, required by geometric techniques, may not be available in some cases. On the other hand, note that in Eq. (12), only the squared magnitude $\vec{e}^{T}[k] \vec{e}[k]$ of the excitation signal is needed. Hence, algebraic methods are employed only when the component-wise reference inputs are not available, as in low-cost in-field calibration. In our work, since the FMS orientation is represented by the matrix $\mathbf{C}_{p}^{q}$, known for each orientation, component-wise reference inputs for accelerometers and magnetometers are available, making it possible to employ geometric techniques for these two sensors.

\subsubsection{Accelerometer parameter estimation by the $L M A$}

Considering its accuracy advantage, we employ a geometric ellipsoid parameter estimation technique that uses the Levenberg-Marquardt algorithm (LMA) [57] to perform nonlinear optimization. The LMA estimates the measurement model parameters of accelerometers according to the model in Eq. (5) by minimizing the fitness (cost) function of the geometric fit described by Eq. (11), given that the excitation signal $\vec{e}=\vec{g}^{\text {NED }}$ is available through calculation (see Eq. (17)). Background information and the notation used for the LMA are provided in Appendix A. To put the accelerometer calibration problem into the framework of the LMA, we need the following definitions:

- We define the fitness function to be minimized by the LMA as $\|\vec{y}-\vec{G}(\vec{\theta})\|$.

- Accelerometer measurements are used to form a single column vector of $3 N$ elements:

$\vec{y}=\left[\begin{array}{llll}\vec{a}_{m}^{T}[1] & \vec{a}_{m}^{T}[2] & \cdots & \vec{a}_{m}^{T}[N]\end{array}\right]^{T}$

Here, $\vec{a}_{m}[k], \quad k=1, \ldots, N$, denotes the measurement vector of accelerometers at time sample $k$.

- Accelerometer measurements, predicted according to the model in Eq. (5) and denoted by $\vec{G}(\vec{\theta})$, are also represented as a vector with $3 N$ elements:

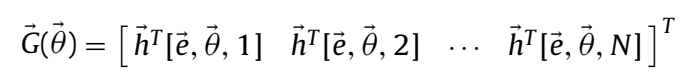

In obtaining $\vec{G}(\vec{\theta}), \mathbf{C}_{p}^{q}[k], k=1, \ldots, N$, which represent the FMS orientations when the FMS is stationary, are used, so that $\vec{e}=\vec{g}^{\mathrm{NED}}$.

- In accordance with $\vec{G}(\vec{\theta})$ and the error components described in Section 2 , the unknown parameter vector $\vec{\theta}$ is given by:

$\vec{\theta}=\left[\begin{array}{lllllllllllll}S_{x} & S_{y} & S_{z} & \alpha_{1} & \alpha_{2} & \alpha_{3} & \epsilon_{x} & \epsilon_{y} & \epsilon_{z} & \beta & b_{x} & b_{y} & b_{z}\end{array}\right]^{T}$

For the ideal sensor that requires no calibration (i.e., with no misalignment, orthogonalization, scale factor, or bias errors), we define the ideal calibration parameter vector, denoted by $\vec{\theta}_{\circ}$, with all its parameters being equal to zero, except for $\alpha_{3}=\pi / 2$, so that:

$\vec{\theta}_{\circ}=\left[\begin{array}{lllllllllllll}0 & 0 & 0 & 0 & 0 & \frac{\pi}{2} & 0 & 0 & 0 & 0 & 0 & 0 & 0\end{array}\right]^{T}$ 
Considering the centrifugal acceleration effects caused by the Earth's rotation, the value of $\vec{g}^{\text {NED }}$ at the location where the experiments are conducted can be calculated using [10]:

$$
\vec{g}^{\mathrm{NED}}=\vec{g}-\frac{(R+\ell)\left\|\vec{\omega}^{\mathrm{NED}}\right\|^{2}}{2}\left[\begin{array}{lll}
\sin 2 \Lambda & 0 & (1+\cos 2 \Lambda)
\end{array}\right]^{T}
$$
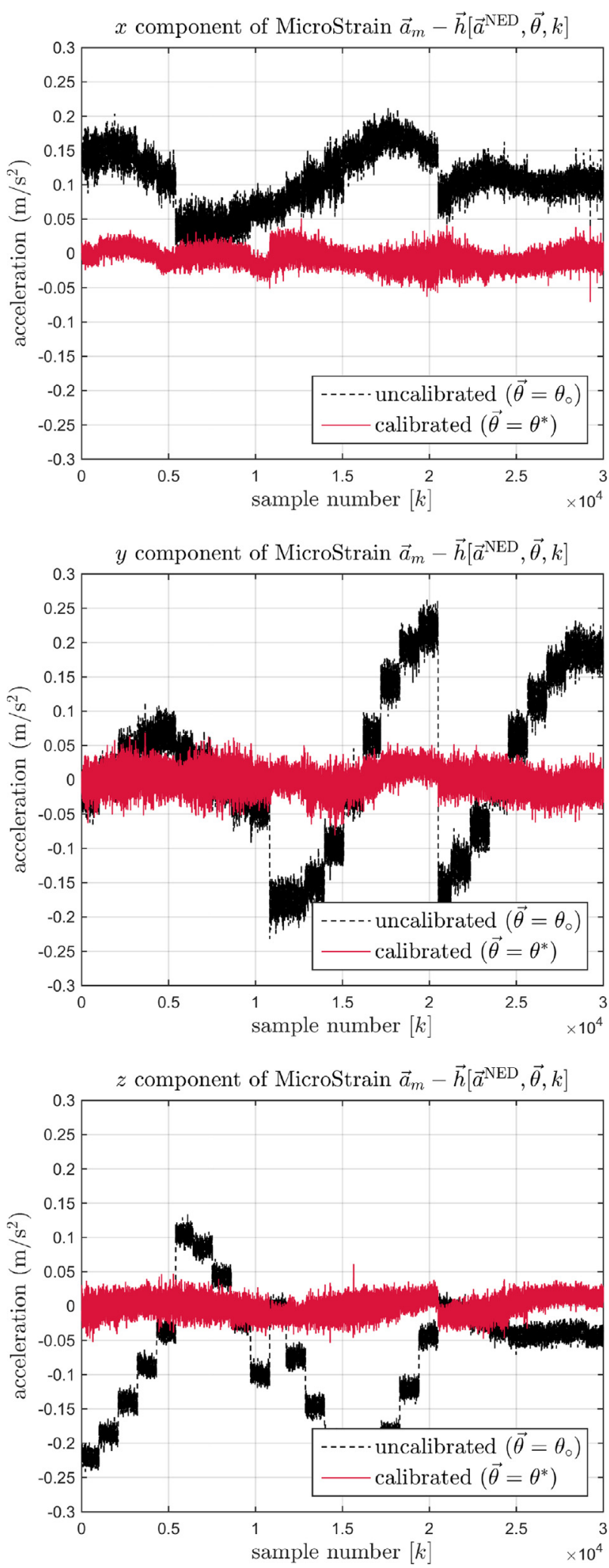

where $\vec{g}=\left[\begin{array}{lll}0 & 0 & 9.80665\end{array}\right]^{T} \mathrm{~m} / \mathrm{s}^{2}$ is the standard gravity vector and $\vec{\omega}^{\mathrm{NED}}, R, \ell$, and $\Lambda$ represent the Earth's angular velocity vector with respect to the NED frame, the radius of the Earth, altitude with respect to sea level, and the latitude angle that changes between $-90^{\circ}$ and $90^{\circ}$, respectively. The calculated $\vec{g}^{\mathrm{NED}}$ vector is given by $\vec{g}^{\mathrm{NED}}=\left[\begin{array}{llll}-0.0167 & 0 & 9.7782\end{array}\right]^{T} \mathrm{~m} / \mathrm{s}^{2}$.
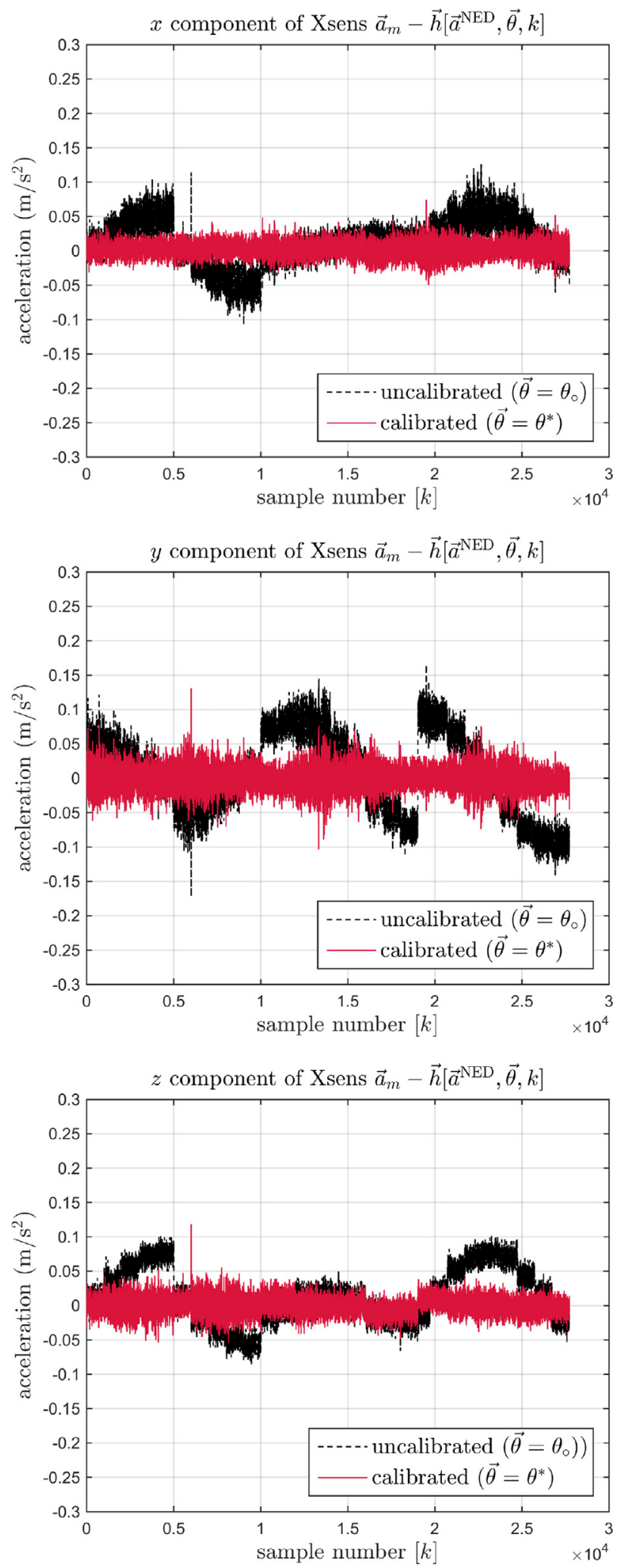

Fig. 6. Comparison of the uncalibrated and calibrated acceleration measurement errors of each axis of the two units. 
Table 1

Measurement errors of the MicroStrain and Xsens accelerometers without and with deterministic calibration. The third and fourth columns provide the results of the Monte-Carlo runs (mean plus/minus one standard deviation) using the traditional and proposed measurement models, respectively.

\begin{tabular}{lccc}
\hline & $\begin{array}{c}\left\|\vec{y}-\vec{G}\left(\vec{\theta}_{\circ}\right)\right\| \\
\left(\mathrm{m} / \mathrm{s}^{2}\right) \\
\text { No calibration }\end{array}$ & $\begin{array}{c}\left\|\vec{y}-\vec{G}\left(\overrightarrow{\theta^{*}}\right)\right\| \\
\left(\mathrm{m} / \mathrm{s}^{2}\right) \\
\text { Traditional }\end{array}$ & $\begin{array}{c}\|\vec{y}-\vec{G}(\vec{\theta})\| \\
\left(\mathrm{m} / \mathrm{s}^{2}\right) \\
\text { Proposed }\end{array}$ \\
\hline MicroStrain & & & \\
Accelerometer- $x$ & 19.02 & $3.41 \pm 0.02$ & $2.87 \pm 0.01$ \\
Accelerometer- $y$ & 20.24 & $3.04 \pm 0.03$ & $2.67 \pm 0.01$ \\
Accelerometer- $z$ & 19.92 & $2.83 \pm 0.02$ & $2.12 \pm 0.02$ \\
Xsens & & & \\
Accelerometer- $x$ & 5.71 & $2.46 \pm 0.03$ & $2.21 \pm 0.02$ \\
Accelerometer- $y$ & 9.48 & $2.76 \pm 0.03$ & $2.52 \pm 0.02$ \\
Accelerometer- $z$ & 6.94 & $2.31 \pm 0.03$ & $1.76 \pm 0.01$ \\
\hline
\end{tabular}

The uncalibrated acceleration measurement errors of the two units are illustrated in Fig. 6 . The $\vec{G}\left(\vec{\theta}_{\circ}\right)$ in the second column of each part of Table 1 is calculated by using the $\vec{\theta}_{\circ}$ in Eq. (16) (i.e., assuming an ideal sensor). Note that since $\vec{h}\left[\vec{g}^{\mathrm{NED}}, \vec{\theta}_{\circ}, k\right]=\vec{g}^{\mathrm{NED}}$ for $k=1, \ldots, N$, the elements of $\vec{G}\left(\vec{\theta}_{\circ}\right)$ in Eq. (14) are considerably simplified in this case. The errors given in the second column of Table 1 correspond to the square root of the sum of the uncalibrated squared errors (the $L^{2}$ norm) in Fig. 6 for each axis of the units.

The acquired observations are processed with the LMA, whose input parameters are selected empirically as follows: $\tau=1$, $\varepsilon_{1}=\varepsilon_{2}=10^{-10}$, and $\vec{\theta}_{\text {init }}$ is randomized using a uniform distribution for each parameter (see Appendix A). Note that the values of the parameters $\varepsilon_{1}$ and $\varepsilon_{2}$ affect the termination of the algorithm. It is observed that the LMA converges to a minimum in $13 \pm 3$ iterations for the accelerometers of both units, each iteration taking about eight seconds. We have performed a Monte-Carlo simulation of 100 runs. The mean and standard deviations of the converged calibration parameters are given in Table 2 . When these parameters are used, substantial improvement is achieved in the model fit and errors are considerably reduced. The errors after calibration are shown in Fig. 6 and given in Table 1. The error values given in the fourth column of each part of Table 1 are the square root of the sum of the calibrated squared errors (the $L^{2}$ norm) in Fig. 6 for each axis of the units, averaged over the 100 runs.

The true value of $\beta$ during the experiments was measured to be $89^{\circ}=1.553 \mathrm{rad}$ using a gyro-theodolite. The estimated $\beta$ values given in Table 2 for the two units are close to the true value. The difference between the two estimates is about $7^{\circ}$, indicating their consistency. We note that the angle $\beta$ is a rotation about the $z$ axis of the NED frame, thereby not affecting the vertical component of the gravity, while the 600-times-smaller horizontal component is affected. The value of the horizontal gravity component is $-0.0167 \mathrm{~m} / \mathrm{s}^{2}$; thus comparable to the noise levels of the

\section{Table 2}

Mean plus/minus one standard deviation of the elements of the calibration parameter vector $\vec{\theta}^{*}$ for the accelerometers of the two units for the improved measurement model proposed in this study.

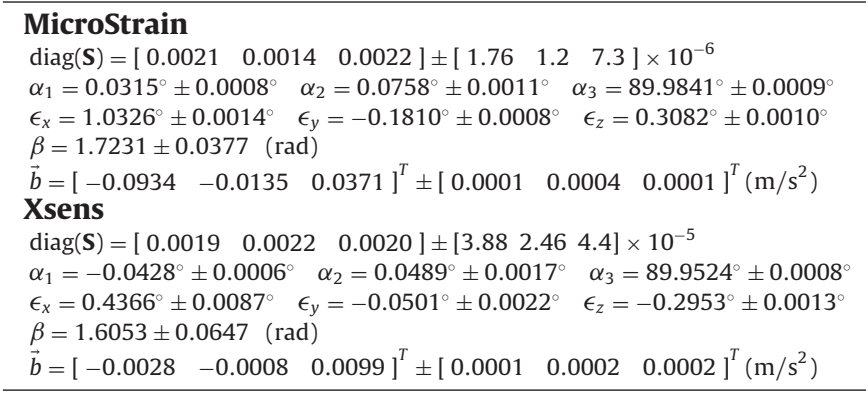

Table 3

Mean plus/minus one standard deviation of the elements of the calibration parameter vector $\vec{\theta}^{*}$ for the accelerometers of the two units according to the traditiona measurement model.

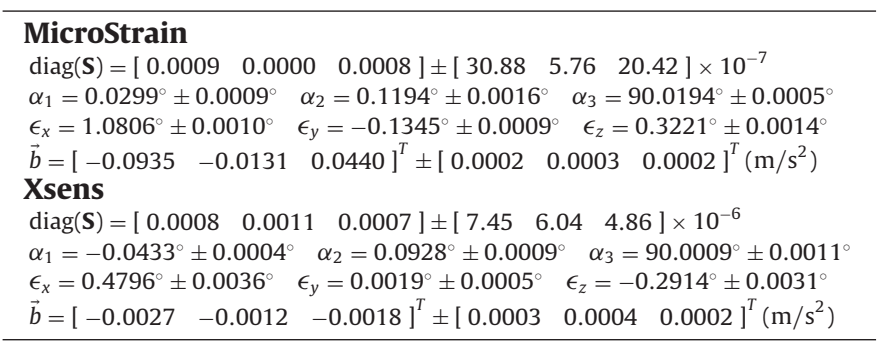

two accelerometers with standard deviations of $0.0072 \mathrm{~m} / \mathrm{s}^{2}$ (for MicroStrain) and $0.0090 \mathrm{~m} / \mathrm{s}^{2}$ (for Xsens). This low signal-to-noise ratio (SNR) makes the estimation of $\beta$ difficult. We have also run the LMA using the true value of $\beta$ and have found the remaining parameter estimates to be very close to those reported in Table 2 .

In our work reported in [21], we used the simpler traditional measurement model for accelerometers given by Eq. (4), where the excitation signal (at stationary positions of the FMS) was taken as $\vec{g}^{p}$ instead of $\vec{g}^{\text {NED }}$. When using the traditional model, we made the following approximation to $\vec{g}^{p}$ : given that the $\vec{i}-\vec{j}$ planes of both $p$ and NED frames lie on the horizontal plane (perpendicular to $\vec{g}$ ) with coincident $\vec{k}$ unit vectors, as shown in Fig. 3(a) and described by Eq. (2), the main component of both $\vec{g}^{p}$ and $\vec{g}^{\text {NED }}$ lies along the $\vec{k}$ direction and the much smaller components along $\vec{i}^{p}, \vec{j}^{p}, \vec{i}^{\mathrm{NED}}$, and $\vec{j}^{\text {NED }}$ can be neglected. Thus, the excitation signal $\vec{g}^{p}$ of that model was approximated by the third component of $\vec{g}^{\text {NED }}$ and used in the calibration procedure since it was not directly available (unlike $\vec{g}^{\mathrm{NED}}$ ). The results obtained by using the traditional model, averaged over the 100 runs, are provided in Table 3 and the third column of Table 1. With the improved measurement model proposed in this study, the errors are reduced by $18 \%$ for the MicroStrain and $14 \%$ for the Xsens accelerometers at the expense of estimating an additional parameter $\beta$. If we use the true value of $\beta$ in the improved model, the error reduction is about the same. Substantial improvement in the model fit would make the stand-alone use of our accelerometers possible for longer durations.

According to the performance measures given in Table 1, it is observed that without any deterministic calibration, the Xsens accelerometer would have better performance than the MicroStrain accelerometer. After calibration, the variation of the Xsens accelerometer measurements around the true values is still lower than the MicroStrain's, although the improvement achieved by deterministic calibration is greater for the MicroStrain than for Xsens.

\subsubsection{Gyroscope parameter estimation by PSO}

The lack of reference angular rates for the FMS and a welldefined model for the error term prevents us from adopting the LMA for gyroscope calibration. Our approach is to use the angular orientation matrix $\mathbf{C}_{p}^{q}$ that the FMS provides as reference and compare it with its estimate obtained by integrating the gyroscope rate measurements. Since the analytical derivation of a closedform expression for the error between the reference and computed angular positions is not an easy task, we relax this difficulty by employing a model-free evolutionary optimization algorithm (PSO) [58].

The angular position of the FMS satisfies the following differential equation:

$\dot{\mathbf{C}}_{p}^{q}=\mathbf{C}_{p}^{q} \mathbf{\Omega}^{q}$ 
where $\Omega^{q}$ is the skew-symmetric matrix generated by the angular rate vector $\vec{\omega}^{q}=\left[\begin{array}{lll}\omega_{x}^{q} & \omega_{y}^{q} & \omega_{z}^{q}\end{array}\right]^{T}$ of frame $q$ with respect to $p[10]$ :

$\boldsymbol{\Omega}^{q}=\left[\begin{array}{ccc}0 & -\omega_{z}^{q} & \omega_{y}^{q} \\ \omega_{z}^{q} & 0 & -\omega_{x}^{q} \\ -\omega_{y}^{q} & \omega_{x}^{q} & 0\end{array}\right]$

The propagation of $\mathbf{C}_{p}^{q}$ between two consecutive time steps $\left(t_{k-1}\right.$ and $t_{k}$ ) is given by [10]:

$\mathbf{C}_{p}^{q}[k]=\mathbf{C}_{p}^{q}[k-1] \exp \left(\int_{t_{k-1}}^{t_{k}} \boldsymbol{\Omega}^{q}(t) d t\right)$

If the sampling interval $\left(T_{s} \triangleq t_{k}-t_{k-1}\right)$ is sufficiently small, Eq. (20) can be approximated by

$\mathbf{C}_{p}^{q}[k]=\mathbf{C}_{p}^{q}[k-1] \exp \left(T_{s} \boldsymbol{\Omega}^{q}[k-1]\right)$

Eqs. (18)-(21) are valid regardless of whether the quantities involved are reference or computed values. In the following, the computed (estimated) values of $\mathbf{C}_{p}^{q}, \boldsymbol{\Omega}^{q}$, and $\vec{\omega}^{q}$ based on gyroscope rate measurements are denoted by $\hat{\mathbf{C}}_{p}^{q}, \hat{\mathbf{\Omega}}^{q}$, and $\hat{\omega}^{q}$, respectively.

To relate the analytical solution of $\mathbf{C}_{p}^{q}[k]$ in Eq. (21) to our gyroscope rate measurements $\vec{\omega}_{m}$, we use the measurement model in Eq. (6) and leave the term $\hat{\vec{\omega}}^{q} \triangleq \hat{\mathbf{C}}_{p}^{q} \vec{\omega}^{p}$ alone on the left-hand side as follows:

$\hat{\hat{\omega}}^{q}=\mathbf{C}_{r}^{q} \mathbf{T}_{s}^{r}(\mathbf{I}+\mathbf{S})^{-1}\left(\vec{\omega}_{m}-\vec{b}\right)$

where $\mathbf{C}_{r}^{q}=\left(\mathbf{C}_{q}^{r}\right)^{-1}=\left(\mathbf{C}_{q}^{r}\right)^{T}$ and $\mathbf{T}_{s}^{r}=\left(\mathbf{T}_{r}^{s}\right)^{-1}$. Since the right-hand side of Eq. (22) contains terms that depend on $\vec{\theta}, \hat{\mathbf{C}}_{p}^{q}$, which depends on $\hat{\hat{\omega}}^{q}$, is also a function of $\vec{\theta}$. Therefore, $\vec{\theta}$ can be included as an argument of $\hat{\mathbf{C}}_{p}^{q}$ as $\hat{\mathbf{C}}_{p}^{q}[\vec{\theta}, k]$, so that the update equation becomes:

$\hat{\mathbf{C}}_{p}^{q}[\vec{\theta}, k]=\hat{\mathbf{C}}_{p}^{q}[\vec{\theta}, k-1] \exp \left(T_{s} \hat{\mathbf{\Omega}}^{q}[\vec{\theta}, k-1]\right)$

Expressing the calibrated gyroscope rate vector as in Eq. (22) allows us to relate $\vec{\omega}_{m}$ and $\hat{\mathbf{C}}_{p}^{q}[\vec{\theta}, k]$ : Once $\hat{\vec{\omega}}^{q}$ is obtained, the matrix $\hat{\boldsymbol{\Omega}}^{q}$ is generated based on this vector using Eq. (19), and then $\hat{\mathbf{C}}_{p}^{q}[\vec{\theta}, k]$ can be computed for a given initial orientation using Eq. (23).

Having obtained an explicit expression for $\hat{\mathbf{C}}_{p}^{q}[\vec{\theta}, k]$, we can define our minimization problem to estimate the calibration parameter vector $\vec{\theta}$. A fitness function $F(\vec{\theta})$ (see Appendix B) is defined as the sum of the squared differences between the elements of $\mathbf{C}_{p}^{q}$ and its estimate $\hat{\mathbf{C}}_{p}^{q}$, which is considered to be the performance criterion:

$F(\vec{\theta})=\sum_{k \in N_{s}}\left\|\mathbf{C}_{p}^{q}[k]-\hat{\mathbf{C}}_{p}^{q}[\vec{\theta}, k]\right\|_{F}$

where $N_{s}$ is the subset of all time instances at which the FMS is

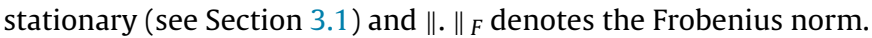

We use PSO to estimate the gyroscope calibration parameters that minimize this fitness function. The parameter vector that PSO optimizes in the search space is the same as in accelerometer calibration except that the vector dimension is reduced by one since the parameter $\beta$ of the vector in Eq. (15) is not included. A brief description of PSO and guidelines for the selection of the configuration parameters can be found in Appendix B and in $[58,59]$. In our implementation, the population size, inertia weight, social, and cognitive parameters are respectively selected as $P=90, m=0.8$, and $\varphi_{p}=\varphi_{g}=2$. Initial positions of the particles are determined randomly in the search space. The $k_{\max }$ and $\Delta_{\max }$ parameters that affect the termination of the PSO are selected as 3000 and 100, respectively.
Table 4

Elements of the calibration parameter vector $\vec{\theta}^{*}$ for the gyroscopes of the two units.

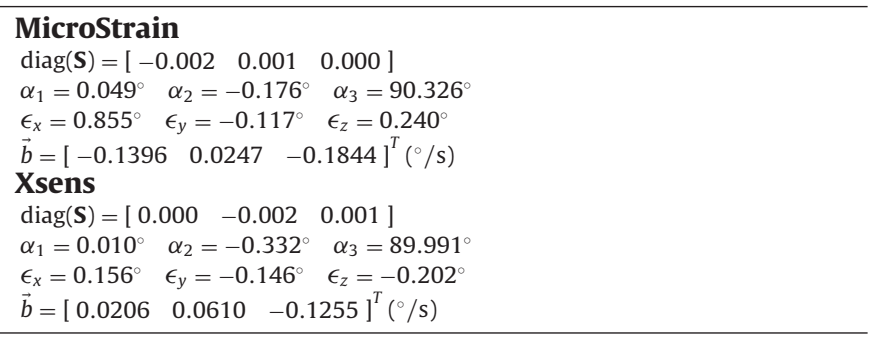

Table 5

Gyroscope measurement errors without and with the calibration of both units.

\begin{tabular}{lcc}
\hline & $F\left(\vec{\theta}_{\circ}\right)$ & $F\left(\vec{\theta}^{*}\right)$ \\
\hline MicroStrain & 12.09 & 0.64 \\
Xsens & 16.91 & 0.68
\end{tabular}

The acquired measurements are processed with PSO, which converges to a minimum in about 827 and 362 iterations for the MicroStrain and Xsens units, respectively. Each iteration takes about $90 \mathrm{~s}$. Elements of the resulting calibration parameter vector, denoted by $\vec{\theta}^{*}$, are provided in Table 4 . The best fitness function values without and with deterministic calibration are given in Table 5 as $F\left(\vec{\theta}_{\circ}\right)$ and $F\left(\vec{\theta}^{*}\right)$, respectively. In calculating $F\left(\vec{\theta}_{\circ}\right)$, the $\vec{\theta}_{\circ}$ given in Eq. (16) for the ideal sensor is used by deleting the fourth zero from the end, which corresponds to the $\beta$ parameter. It can be stated that a significant reduction in attitude error is achieved for both types of units as a result of deterministic calibration. In contrast to accelerometers, the MicroStrain gyroscope has better error characteristics than Xsens in terms of accuracy, for both the uncalibrated and calibrated cases.

An important issue in applications requiring precise attitude estimation is achieving convergence to a globally optimal calibration parameter vector $\vec{\theta}^{*}$ rather than to a locally optimal one. The fitness function defined in Eq. (24) corresponds to the sum of the errors between the outputs of first-order difference equations (see Eq. (23)) and is more complicated compared to the fitness functions in off-field calibration, which are typically composed of ordinary momentary measurement equations (e.g., Eq. (14)). To our knowledge, this is the first study to implement a global optimization algorithm for this specific problem. With our proposed methodology, we show that low-cost calibration techniques become sufficiently reliable and provide fair error reduction without the use of any special rate-controlled machine. Our gyroscope calibration technique does not require precise angular velocity information but employs reference angular positions. This does not necessarily require costly equipment and can even be realized with a simple mechanical structure such as a right-angled fixture plate onto which gyroscopes are mounted. The fixture plate is then rotated by hand on a flat surface to obtain reference angular positions. Since we employ an FMS, we do not position gyroscopes by hand movements in this study. However, calibration parameters are estimated similarly to an experiment based on arbitrary rotations by hand, thus making our approach suitable for low-cost in-field gyroscope calibration. Finally, we note that for in-field calibration, the PSO technique for parameter estimation would have to be replaced by a faster algorithm.

\subsubsection{Magnetometer parameter estimation by the $L M A$}

The magnetometer measurement model is more complicated than the inertial sensor models since the soft- and hard-iron errors, Kand $\delta \vec{B}$, are involved, in addition to the error components included in the inertial sensor measurement models [Eqs. (5) and (6)]. 
Despite these additional error types, inertial sensors and magnetometers can be represented by the unified model given in Eq. (9). Accelerometers and magnetometers also share something in common in terms of component-wise reference data availability: $\vec{B}^{\text {NED }}$ at the location where the experiments are conducted can be looked up, as in the accelerometer case where $\vec{g}^{\mathrm{NED}}$ can be calculated. Regarding these similarities, the same approach for accelerometers can be followed here to estimate the parameters needed for magnetometer calibration. Therefore, we use the LMA to minimize the calibration error, given in Eq. (11), in magnetometer measurements.

We investigate magnetometer calibration in two parts: First, we consider a traditional magnetometer measurement model and estimate its parameters using the LMA. Then, we extend the measurement model considering the external magnetic disturbances our magnetometers are exposed to and show its efficacy by repeating the parameter estimation procedure.

The following definitions express our minimization problem according to the LMA terminology and are valid for both the traditional and proposed models:

- The fitness function to be minimized by the LMA is given by $\| \vec{y}-$ $\vec{G}(\vec{\theta}) \|$, as in the accelerometer case.

- The vector $\vec{y}$ is formed by arranging the measurement vectors as

$\vec{y}=\left[\begin{array}{llll}\vec{B}_{m}^{T}[1] & \vec{B}_{m}^{T}[2] & \cdots & \vec{B}_{m}^{T}[N]\end{array}\right]^{T}$

where $\vec{B}_{m}[k], \quad k=1, \ldots, N$, denotes the measurement vector of the magnetometer at time sample $k$.

- $\vec{G}(\vec{\theta})$ can be expressed in a way similar to Eq. (14) if $\vec{h}[\vec{e}, \vec{\theta}, k]$ is determined as in Eq. (7):

$$
\vec{G}(\vec{\theta})=\left[\begin{array}{llll}
\vec{h}^{T}\left[\vec{B}^{\mathrm{NED}}, \vec{\theta}, 1\right] & \vec{h}^{T}\left[\vec{B}^{\mathrm{NED}}, \vec{\theta}, 2\right] & \ldots & \left.\vec{h}^{T}\left[\vec{B}^{\mathrm{NED}}, \vec{\theta}, N\right]\right]^{T}
\end{array}\right.
$$

In obtaining $\vec{G}(\vec{\theta})$, again we use the $\mathbf{C}_{p}^{q}[k], \quad k=1, \ldots, N$, which represent the FMS orientations when the FMS is stationary.

The value of $\vec{B}^{\mathrm{NED}}$ at the facility where the experiments are conducted is looked up from the World Magnetic Model 2015 [60] and found to be $\vec{B}^{\mathrm{NED}}=\left[\begin{array}{lll}0.2523 & 0.0217 & 0.4004\end{array}\right]^{T}$ Gauss. Note that the small component in the East direction is because of the magnetic declination angle between the true North and the magnetic North.

Component-wise raw magnetometer measurements acquired from each axis of the two units are presented in Figs. 7(a) and (b). We observe that these are highly corrupted and asymmetric. In Fig. 7(c), we provide the reference magnetic field vector magnitude and the magnitude calculated using the measurements in parts (a) and (b), between which we observe large deviations. Modeling such large deviations requires knowledge of $\mathbf{C}_{\mathrm{NED}}^{p}$, since ideal magnetometer measurements can be obtained by mapping $\vec{B}^{\mathrm{NED}}$ onto the sensor enclosure frame $q$, via sequential transformations from NED to $p$ and then from $p$ to $q$ frames. Although the latter transformation matrix $\mathbf{C}_{p}^{q}$ is known, the parameter $\beta$ involved in $\mathbf{C}_{\mathrm{NED}}^{p}$, given in $\mathrm{Eq}$. (2), needs to be estimated.

3.2.3.1. Traditional model-based calibration. We consider the traditional magnetometer measurement model given in Eq. (7). In the unified measurement model of Eq. (8), the errors $\delta \vec{B}$ and $\vec{B}$ were combined into a unified bias vector $\vec{b}^{\prime}=(\mathbf{I}+\mathbf{S}) \mathbf{T}_{r}^{S} \mathbf{C}_{q}^{r} \delta \vec{B}+\vec{b}$. Therefore, this model contains 19 unknown parameters (three parameters in $\mathbf{S}, \mathbf{T}_{r}^{s}$, and $\mathbf{C}_{q}^{r}$ each, six in $\mathbf{K}$, one in $\mathbf{C}_{\mathrm{NED}}^{p}$, and three in $\vec{b}^{\prime}$ ).
Table 6

Measurement errors of the MicroStrain and Xsens magnetometers without and with deterministic calibration. The third and fourth columns provide the results of the Monte-Carlo runs (mean plus/minus one standard deviation) using the traditional and proposed measurement models, respectively.

\begin{tabular}{lccc}
\hline & $\begin{array}{c}\left\|\vec{y}-\vec{G}\left(\vec{\theta}_{\circ}\right)\right\| \\
\text { (Gauss) } \\
\text { No calibration }\end{array}$ & $\begin{array}{c}\left\|\vec{y}-\vec{G}\left(\vec{\theta}^{*}\right)\right\| \\
\text { (Gauss) } \\
\text { Traditional }\end{array}$ & $\begin{array}{c}\left\|\vec{y}-\vec{G}\left(\vec{\theta}^{*}\right)\right\| \\
\text { (Gauss) } \\
\text { Proposed }\end{array}$ \\
\hline $\begin{array}{l}\text { MicroStrain } \\
\text { magnetometer- } x\end{array}$ & 17.7 & $9.281 \pm 0.116$ & $6.409 \pm 0.044$ \\
magnetometer- $y$ & 29.6 & $19.502 \pm 0.167$ & $6.486 \pm 0.071$ \\
magnetometer- $z$ & 67.5 & $14.643 \pm 0.073$ & $7.601 \pm 0.102$ \\
Xsens & 148 & $18.331 \pm 0.240$ & $8.446 \pm 0.016$ \\
magnetometer- $x$ & 134 & $22.904 \pm 0.118$ & $12.573 \pm 0.094$ \\
magnetometer- $y$ & 151 & $30.319 \pm 0.277$ & $10.412 \pm 0.106$ \\
magnetometer- $z$ & & & \\
\hline
\end{tabular}

Defining $\mathbf{W} \triangleq(\mathbf{I}+\mathbf{S}) \mathbf{T}_{r}^{s} \mathbf{C}_{q}^{r} \mathbf{K}$, it follows that $\mathbf{H}=\mathbf{W} \mathbf{C}_{p}^{q} \mathbf{C}_{\mathrm{NED}}^{p}$, and we can rewrite the measurement model in Eq. (7) according to the unified measurement model as follows:

$\vec{B}_{m}=\mathbf{W} \mathbf{C}_{p}^{q} \mathbf{C}_{\mathrm{NED}}^{p} \vec{B}^{\mathrm{NED}}+\vec{b}^{\prime}+\vec{n}$

We observe that $\mathbf{W}$ is a function of 15 parameters (three parameters in $\mathbf{S}, \mathbf{T}_{r}^{s}$, and $\mathbf{C}_{q}^{r}$ each, and six in $\left.\mathbf{K}\right)$. Given that the $\mathbf{W}$ matrix contains nine parameters corresponding to each one of its elements $w_{i j}$ where $i, j=1,2,3$, at most nine of these 15 parameters can be independent. It is not possible to observe and estimate all of the underlying 15 parameters. Since $\mathbf{C}_{\mathrm{NED}}^{p}$ is a function of $\beta$, and $\vec{b}^{\prime}$ also contains three parameters, 13 parameters in total are involved in this measurement model. An observability analysis [61], similar to that in [62], shows that all of these 13 parameters can be observed through the set of measurement equations. We therefore limit the size of our parameter vector to 13 , as follows:

$\vec{\theta}=\left[\begin{array}{lllllllllllll}w_{11} & w_{12} & w_{13} & w_{21} & w_{22} & w_{23} & w_{31} & w_{32} & w_{33} & \beta & b_{x}^{\prime} & b_{y}^{\prime} & b_{z}^{\prime}\end{array}\right]^{T}$

For the ideal sensor that does not require calibration (without any type of error), $\mathbf{W}=\mathbf{I}_{3 \times 3}$ and the calibration parameter vector $\vec{\theta}_{\circ}$ simplifies to:

$\vec{\theta}_{\circ}=\left[\begin{array}{lllllllllllll}1 & 0 & 0 & 0 & 1 & 0 & 0 & 0 & 1 & 0 & 0 & 0 & 0\end{array}\right]^{T}$

Measurement errors without calibration are given in the second column of Table 6 . The $\vec{G}\left(\vec{\theta}_{\rho}\right)$ here is calculated using the $\vec{\theta}_{\circ}$ given in Eq. (29). Note that since $\vec{h}\left[\vec{B}^{\mathrm{NED}}, \vec{\theta}_{\circ}, k\right]=\vec{B}^{\mathrm{NED}}$ for $k=1, \ldots, N$, the elements of $\vec{G}\left(\vec{\theta}_{\circ}\right)$ in Eq. (26) are considerably simplified in this case.

We run the LMA to estimate the magnetometer calibration parameters. The configuration parameters, initialization of the parameter vector $\vec{\theta}_{\text {init }}$, and the termination conditions of the LMA are the same as in estimating the measurement model parameters of accelerometers. The algorithm converges in $12 \pm 3$ iterations and each iteration takes about $10 \mathrm{~s}$. The mean and standard deviations of the parameter values (over the 100 Monte-Carlo runs) estimated by the LMA are given in Table 7 . The poor estimation of $\beta$ might have been caused by the low SNR and inadequate modeling of the errors. Although the residual errors, given in the third column of Table 6, are reduced after calibration, percentage-wise reduction is not as much as that of inertial sensors, despite that the noise levels of accelerometers and magnetometers are comparable. The accuracy of the parameter estimates and the amount of error may not be acceptable for certain applications. When the dataset is carefully examined, a time-varying component is observed on the measurements. Such time-varying behavior has been usually attributed to temperature-dependent bias error [6]. However, temperature cannot be the reason in this case since the operating temperature of 


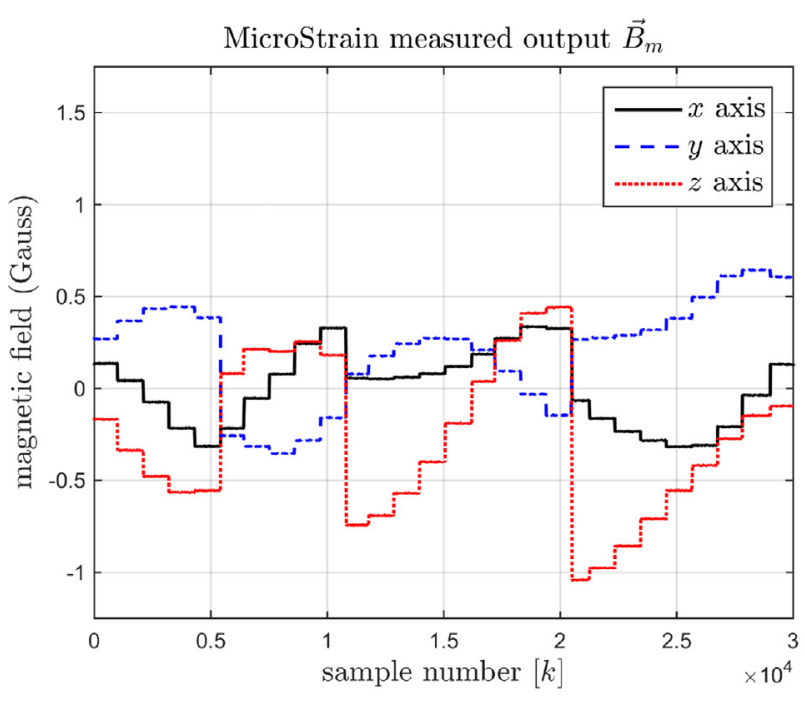

(a)

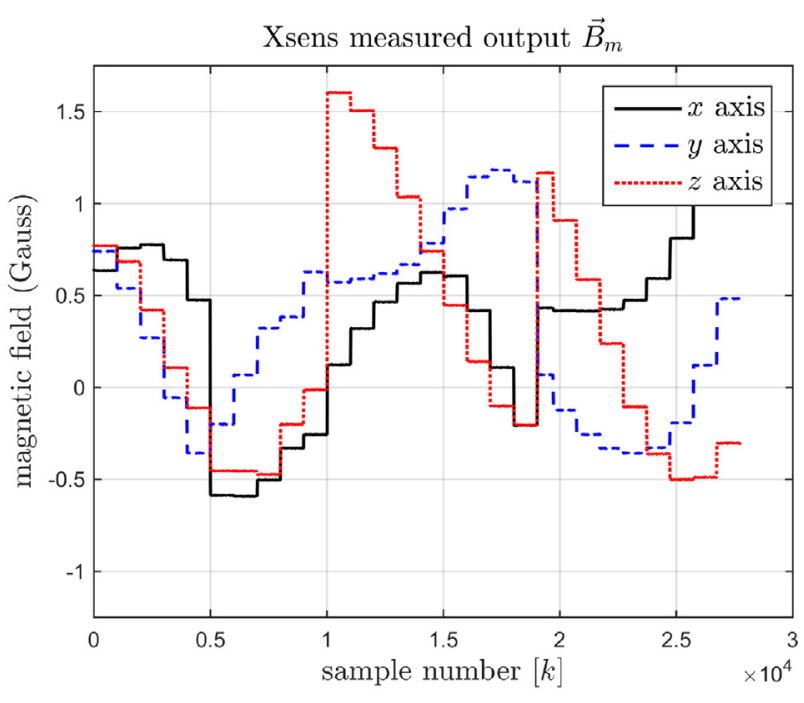

(b)

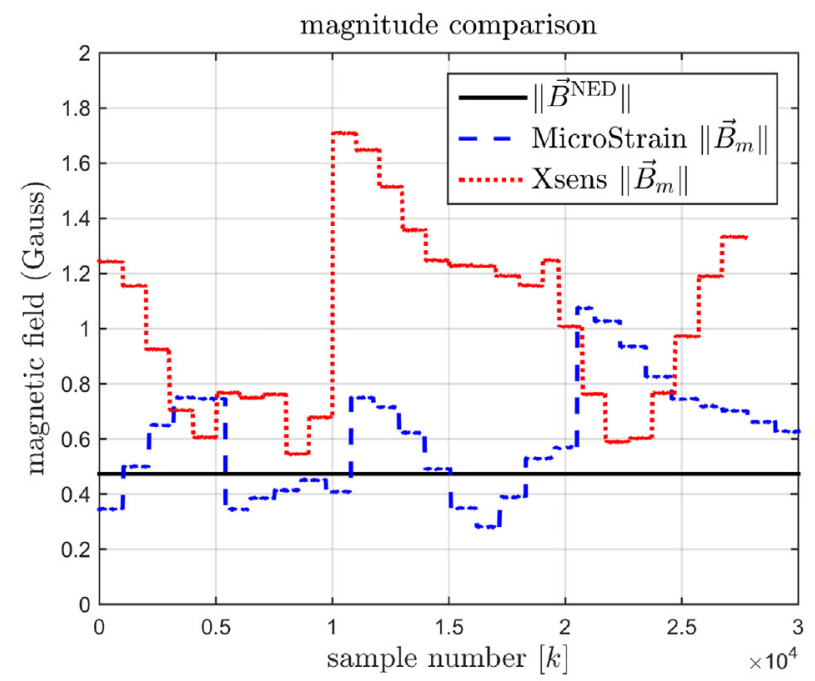

(c)

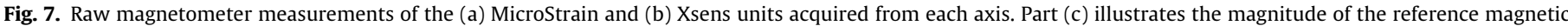
field vector and the magnitude calculated using the measurements in (a) and (b) for each unit.

the sensors does not change significantly given the short duration of the experiments.

3.2.3.2. Proposed model-based calibration. We believe that the relatively large residual errors after calibration are caused by the

Table 7

Mean plus/minus one standard deviation of the elements of the calibration parameter vector $\vec{\theta}^{*}$ for the magnetometers of the two units for the traditional model.

\begin{tabular}{|c|c|c|c|c|c|c|c|}
\hline \multicolumn{8}{|c|}{ MicroStrain } \\
\hline & 0.8008 & -0.0903 & $30.1701]$ & & 0.1240 & 0.0827 & $0.0485]$ \\
\hline $\mathbf{W}=$ & -0.3515 & 0.8102 & 0.2521 & \pm & 0.0671 & 0.1054 & 0.0441 \\
\hline \multicolumn{8}{|c|}{$\beta=0.4016 \pm 0.0957(\mathrm{rad})$} \\
\hline \multicolumn{8}{|c|}{ 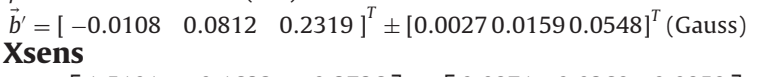 } \\
\hline & {$[1.5101$} & -0.1632 & $-0.3736]$ & & 0.0871 & 0.0369 & $0.0859]$ \\
\hline \multirow[t]{2}{*}{$\mathbf{W}=$} & 0.3294 & 1.8065 & -0.4972 & \pm & 0.0206 & 0.1794 & 0.0746 \\
\hline & 1.1314 & 1.2019 & 2.0297 & & 0.0524 & 0.1083 & 0.1116 \\
\hline \multicolumn{8}{|c|}{$\beta=0.3948 \pm 0.1081(\mathrm{rad})$} \\
\hline \multicolumn{8}{|c|}{$\vec{b}^{\prime}=\left[\begin{array}{lll}0.1215 & 0.2377 & 0.1742\end{array}\right]^{T} \pm\left[\begin{array}{lll}0.0942 & 0.0749 & 0.0510\end{array}\right]^{T}$ (Gauss) } \\
\hline
\end{tabular}

orientation-dependent hard-iron errors that should be accounted for in the measurement model. To test our claim, we propose an extended measurement model for magnetometers that incorporates these effects by transforming the constant hard-iron errors $\delta \vec{B}$ in the non-rotating $p$ frame to the rotating sensor enclosure frame $q$ by pre-multiplying $\delta \vec{B}$ with $\mathbf{C}_{p}^{q}$ as:

$$
\vec{B}_{m}=(\mathbf{I}+\mathbf{S}) \mathbf{T}_{r}^{S} \mathbf{C}_{q}^{r}\left(\mathbf{K} \mathbf{C}_{p}^{q} \mathbf{C}_{\mathrm{NED}}^{p} \vec{B}^{\mathrm{NED}}+\mathbf{C}_{p}^{q} \delta \vec{B}\right)+\vec{b}+\vec{n}
$$

Since the resultant effect of hard-iron errors on the measurements now changes with $\mathbf{C}_{q}^{r} \mathbf{C}_{p}^{q}$ and since $\mathbf{C}_{p}^{q}$ is dependent on the orientation of the FMS, we now consider the $\delta \vec{B}$ and the $\vec{B}$ (which were unified in the traditional calibration model) as separate parameters. Defining $\mathbf{V} \triangleq(\mathbf{I}+\mathbf{S}) \mathbf{T}_{r}^{s} \mathbf{C}_{q}^{r}$, we can rewrite the above equation as

$$
\vec{B}_{m}=\mathbf{V K C} \mathbf{C}_{p}^{q} \mathbf{C}_{\mathrm{NED}}^{p} \vec{B}^{\mathrm{NED}}+\mathbf{V C}_{p}^{q} \delta \vec{B}+\vec{b}+\vec{n}
$$

We express $\mathbf{W}$ as $\mathbf{W}=\mathbf{V K}$, and after noting that $\mathbf{K}$ is symmetric $\left(\mathbf{K}=\mathbf{K}^{T}\right)$, we determine that $\mathbf{V}=\mathbf{W K}^{-1}$ where $\mathbf{K}^{-1}$ is also a symmetric 
Table 8

Mean plus/minus one standard deviation of the elements of the calibration parameter vector $\vec{\theta}^{*}$ for the magnetometers of the two units for the proposed model.

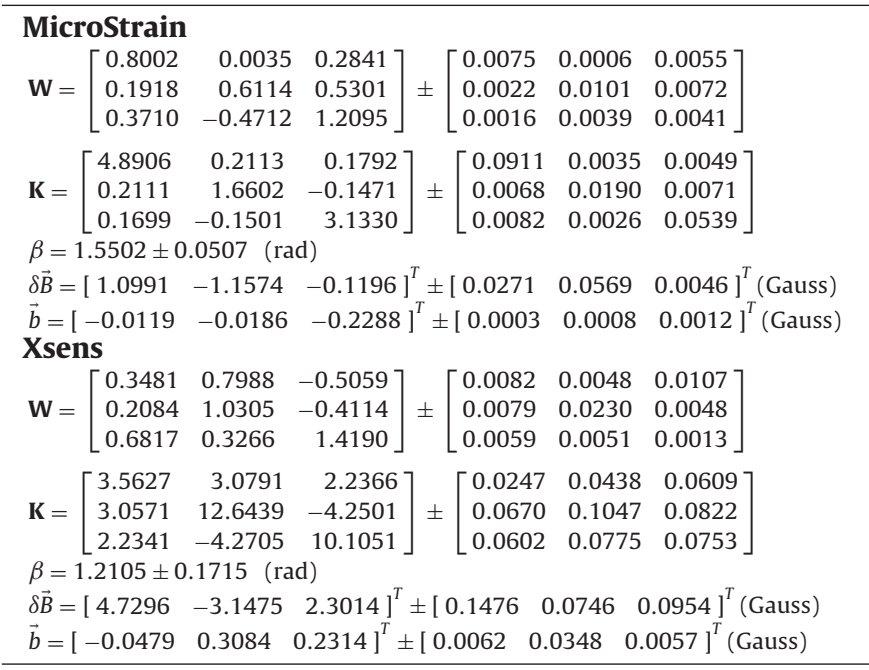

matrix since $\mathbf{K}^{-1}=\left(\mathbf{K}^{T}\right)^{-1}=\left(\mathbf{K}^{-1}\right)^{T}$. We reformulate Eq. (31) based on these definitions as

$\vec{B}_{m}=\mathbf{W C}_{p}^{q} \mathbf{C}_{\mathrm{NED}}^{p} \vec{B}^{\mathrm{NED}}+\mathbf{W K}^{-1} \mathbf{C}_{p}^{q} \delta \vec{B}+\vec{b}+\vec{n}$

Considering the elements of $\mathbf{W}$ and $\mathbf{K}$, the corresponding parameter vector $\vec{\theta}$ is as follows:

$\vec{\theta}=\left[\begin{array}{lllllllllll}w_{11} & w_{12} & w_{13} & w_{21} & w_{22} & w_{23} & w_{31} & w_{32} & w_{33} & k_{11} & k_{12}\end{array}\right.$

An observability analysis shows that all of these 22 parameters are observable through the set of measurement equations [61]. The ideal parameter vector for this case is given by:

$\vec{\theta}_{\circ}=\left[\begin{array}{llllllllllllllllllllll}1 & 0 & 0 & 0 & 1 & 0 & 0 & 0 & 1 & 1 & 0 & 0 & 1 & 0 & 1 & 0 & 0 & 0 & 0 & 0 & 0 & 0\end{array}\right]^{T}$

since both $\mathbf{W}$ and $\mathbf{K}$ become equal to $\mathbf{I}_{3 \times 3}$ and the remaining parameters are ideally zero. We note here that when we use this vector to calculate $\vec{G}\left(\vec{\theta}_{\circ}\right)$, since $\vec{h}\left[\vec{B}^{\mathrm{NED}}, \vec{\theta}_{\circ}, k\right]=\vec{B}^{\mathrm{NED}}$ for $k=1, \ldots, N$, again, the results are no different than those for the traditional model. Therefore, the errors without calibration, given in the second column of Table 6, are valid for both the traditional and proposed models.

We run the LMA for the proposed model, where it converges in $130 \pm 20$ iterations for both units, each iteration taking about $30 \mathrm{~s}$. The mean and standard deviation values of the estimated parameter values (over the 100 Monte-Carlo runs) are given in Table 8. We observe that the $\beta$ estimates given in the table are now much closer to the true value of $1.553 \mathrm{rad}$ (provided in Section 3.2.1), especially so for the MicroStrain unit. The discrepancy between the estimated $\beta$ values of the two magnetometers is about $23.5^{\circ}$ for the proposed model.

The mean residual errors according to the model in Eq. (30) are given in the fourth column of Table 6. Comparing these with the mean residual errors in the third column of the same table, corresponding to the traditional model in Eq. (7), we observe that the errors obtained with the proposed magnetometer model are considerably reduced (by a factor of 1.45-3.05). A comparison of the errors after calibration using the traditional and proposed models is also illustrated in Fig. 8. We also observe that the residual errors of the MicroStrain magnetometer are significantly lower than those of Xsens. Although the proposed model improves the calibration accuracy for both units to some extent, it seems to better capture the underlying measurement errors of the MicroStrain magnetometer.
Consequently, the estimated $\beta$ for the MicroStrain magnetometer is closer to the true $\beta$ value.

The improvement in the errors verifies our claim that orientation-dependence for hard-iron errors should be incorporated in the model when magnetometers are calibrated with test set-ups traditionally employed for inertial sensors (e.g., highDoF rate tables, motion simulators, and robotic arms). The model presented in Eq. (30) makes these set-ups more suitable for magnetometer modeling and calibration. However, the residual errors are still larger than those of inertial sensors, thus we cannot consider the calibration as accurate and reliable as we would like. Despite that the errors are substantially reduced as a result of using the proposed model, perhaps our proposed model does not sufficiently capture and model all of the measurement errors involved. Although the proposed model brings some improvement to calibration accuracy by modeling orientation-dependent hard-iron errors, one should consider it as a base upon which more precise and complex models can be developed.

An interesting possible further extension to this model would be to represent the dependence between hard-iron errors and electric motor currents and other current carrying wires or electronics near the platform in order to eliminate the remaining calibration errors. The results of a somewhat similar dependence are given in [49], in which a significant improvement is achieved in calibration accuracy with the association of the bias terms and the total current drawn from the on-board batteries of a satellite. Considering the need for further improvement to our proposed model for

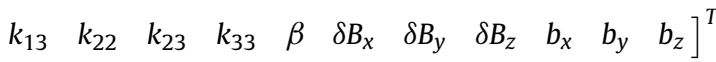

calibration when active angular positioning machines are employed, our extended magnetometer model would be especially useful when passive mechanical positioning structures (e.g., simple right-angled fixture plates) are used to position the sensors during data acquisition. Calibration with such structures is often not sufficiently accurate since the variable effect of constant magnetic distortions in the vicinity of the sensors as the plate is rotated is not considered in traditional calibration models. Our proposed model opens up the possibility to calibrate magnetometers more accurately with such simple, purely mechanical structures, since magnetic disturbances can now be compensated for by the orientation-dependent hard-iron error terms included in the model.

\section{Conclusions and future work}

We addressed the deterministic error modeling and calibration of inertial sensors and magnetometers and estimation of their calibration parameters. We formulated the deterministic measurement model of the sensors based on the results of earlier works. Then, we estimated the measurement model parameters with experimentally acquired data during the calibration procedure using nonlinear optimization algorithms. We proposed an improvement to the traditional measurement model of accelerometers, through which the residual errors obtained by LMA were reduced compared to those of the traditional model. The traditional approach is not suitable for gyroscopes because of the lack of reference angular rates for the FMS used in the experiments. Instead, we developed a technique based on comparing the computed attitude with the reference attitude that the FMS provides. We employed a model-free evolutionary optimization technique, PSO, since the analytical derivation of the attitude error as a function of the sensor calibration parameters would have been a highly 

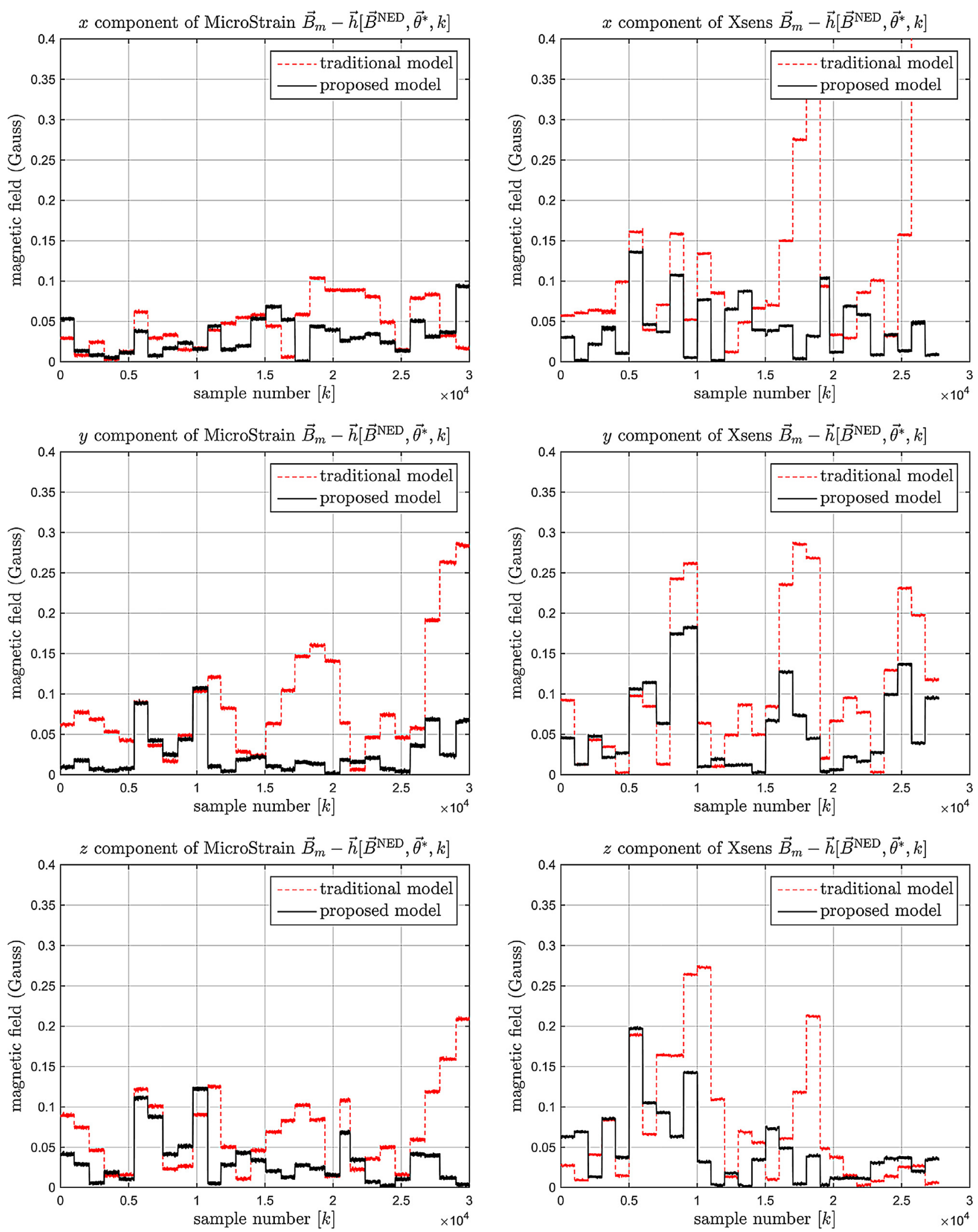

Fig. 8. Calibrated magnetometer measurement errors of each axis of the two units for the traditional and proposed models.

complex task. We have achieved sufficient modeling accuracy with the proposed approach, which is low cost and can also be adapted to in-field calibration. Calibration based on using the traditional model for magnetometers led to unsatisfactory results because of the unmodeled effects (orientation-dependent hard-iron errors). Hence, we proposed an extended sensor model for magnetometers that improved the residual calibration errors by a factor of between 1.45 and 3.05 . 
In future work, the improved model for accelerometers proposed in this study could be extended to handle complete 3D angular misalignments between the NED frame and the platform base frame $p$. This could be done by modifying the $\mathbf{C}_{\mathrm{NED}}^{p}$ matrix so that it represents an arbitrary rotational transformation, involving three parameters that correspond to Euler angles, instead of a single rotational angle $\beta$ about the local vertical direction. The calibration method proposed for gyroscopes could be implemented in a real in-field calibration scenario and compared to the existing techniques. Calibrated sensors could be tested for attitude estimation and navigation in stand-alone and aided IMUs. The magnetometer measurement model could be developed to further improve the calibration accuracy.

\section{Acknowledgments}

The authors are grateful to ROKETSAN Inc. for providing the ACUTRONIC FMS and the facilities to conduct the experiments in. We also thank our colleagues Derya Ünsal and Can Taşan for helping conduct the experiments with the FMS. The two sensor units were purchased with the support of The Scientific and Technological Research Council of Turkey (TÜBITAK) under grant number EEEAG109E059, which participated in MOVE (COST Action IC0903).

\section{Appendix A. Levenberg-Marquardt algorithm (LMA)}

The LMA is an iterative nonlinear optimization technique widely used in a broad range of applications. Let $\vec{y}$ denote the true (measured) output and $\vec{G}(\vec{\theta})$ denote the estimated output vector of a given function in parametric form, where $\vec{\theta}$ is an unknown parameter vector. The function intends to model the relation between the true output and the input that corresponds to it in terms of the unknown parameters. The LMA tries to estimate the optimal parameter vector $\vec{\theta}^{*}$ by minimizing the sum of the squared distances between the true output vector and the estimated value of the function:

$\vec{\theta}^{*}=\underset{\vec{\theta}}{\operatorname{argmin}}|| \vec{y}-\vec{G}(\vec{\theta})||$

The LMA is considered to be a hybrid of the steepest-descent and Gauss-Newton methods [63]. It converges to the minimum by updating the current solution vector $\vec{\theta}$ through a series of augmented least-squares problems formed after the linearization of $\vec{G}(\vec{\theta})$ in the neighborhood of $\vec{\theta}$. This process is detailed below:

- $\vec{G}(\vec{\theta})$ is linearized around $\vec{\theta}$ by making a first-order approximation that neglects the second- and higher-order terms of its Taylor series expansion:

$$
\vec{G}(\vec{\theta}+\delta \vec{\theta}) \cong \vec{G}(\vec{\theta})+\frac{\partial \vec{G}(\vec{\theta})}{\partial \vec{\theta}} \delta \vec{\theta}
$$

- Conventional normal equations related to Eq. (36) can be formed by:

$$
\mathbf{J}^{T} \mathbf{J} \delta \vec{\theta}=\mathbf{J}^{T}[\vec{y}-\vec{G}(\vec{\theta})] \quad \text { where } \mathbf{J}=\frac{\partial \vec{G}(\vec{\theta})}{\partial \vec{\theta}}
$$

- The LMA solves a slightly different form of Eq. (37), which is called the augmented normal equations:

$$
\delta \vec{\theta}=\left(\mathbf{J}^{T} \mathbf{J}+\mu \mathbf{I}\right)^{-1} \mathbf{J}^{T}[\vec{y}-\vec{G}(\vec{\theta})]
$$

where the parameter $\mu$ denotes the scalar damping coefficient of the LMA, which is updated at each iteration. Once $\delta \vec{\theta}$ is obtained, $\vec{\theta}$ is updated according to $\vec{\theta}=\vec{\theta}+\delta \vec{\theta}$.

- The LMA stops if any of the following occurs:
- the preset upper bound for the number of iterations is reached,

- the solution cannot be improved sufficiently,

- the fitting error drops below a certain level.

The pseudo-code of the LMA is given below [63]. The input parameters $\tau, \varepsilon_{1}$, and $\varepsilon_{2}$ are related to the configuration settings used to provide a proper LMA operation. The parameter $\tau$ is related to the initialization of the algorithm, whereas $\varepsilon_{1}$ and $\varepsilon_{2}$ are the termination condition parameters. The choice of $\tau$ depends on the quality of the initial parameter guess $\vec{\theta}_{\text {init }}$. As a rule of thumb, a small value (e.g., $10^{-6}$ ) is used if $\vec{\theta}_{\text {init }}$ is believed to be a fair initial guess. Otherwise, $\tau$ should be set to a value around one. Furthermore, $\varepsilon_{1}$ and $\varepsilon_{2}$ are generally set to very small values such as $10^{-10}$ to guarantee convergence. A more detailed discussion pertaining to the LMA can be found in $[57,63]$.

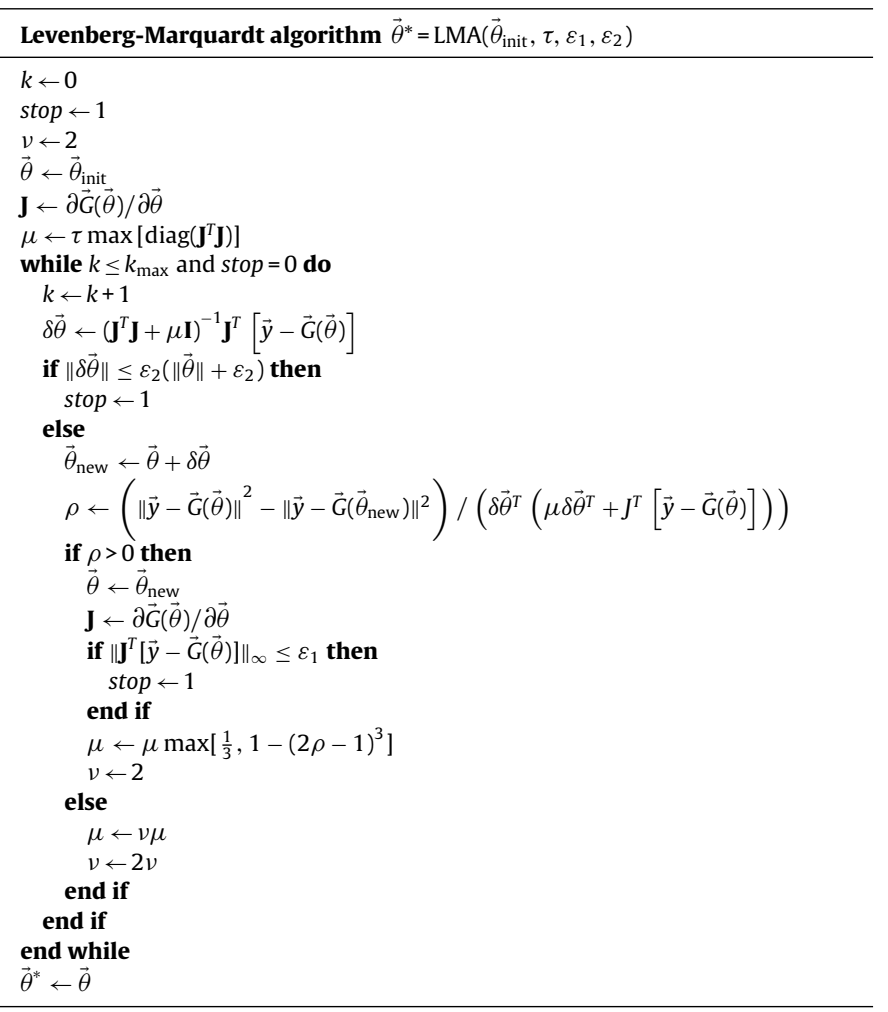

When adopting the LMA for the deterministic calibration of inertial sensors and magnetometers, $\vec{G}(\vec{\theta})$ is selected according to one of the sensor measurement models [Eqs. (5)-(7)]. Then, $\vec{\theta}$ is the set of unknown calibration parameters involved in the model(i.e., parameters related to misalignment, orthogonalization, scale factor, bias, soft- and hard-iron errors).

\section{Appendix B. Particle swarm optimization (PSO)}

Though PSO was originally proposed to simulate movements of bird flocks, it has been increasingly used in optimization problems in many areas, including swarm intelligence, evolutionary programming and computation, signal processing, and sensor calibration [64-66] because of the simplicity of its implementation and its known success in efficient convergence to global extrema [58]. It is generally adopted as an off-line optimization tool since it requires a considerable amount of computational power. The workflow of the algorithm can be described roughly as the search for particles in the parameter space towards the optimal solution. Optimality is measured according to a quality index, described as the fitness (cost) function $F($.$) , that can be either minimized or maximized. The$ general PSO problem is portrayed below: 
Let $P$ be the number of particles in the swarm, the $i$ th of which has a position $\vec{\theta}_{i}$ and a velocity $\vec{v}_{i}$ in the search-space $\mathbb{R}^{d}$. Let $\vec{p}_{i}$ be the best-known position of that particle and $\vec{\theta}^{*}$ be the best-known position of the entire swarm. The pseudo-code of the basic PSO algorithm is as follows:

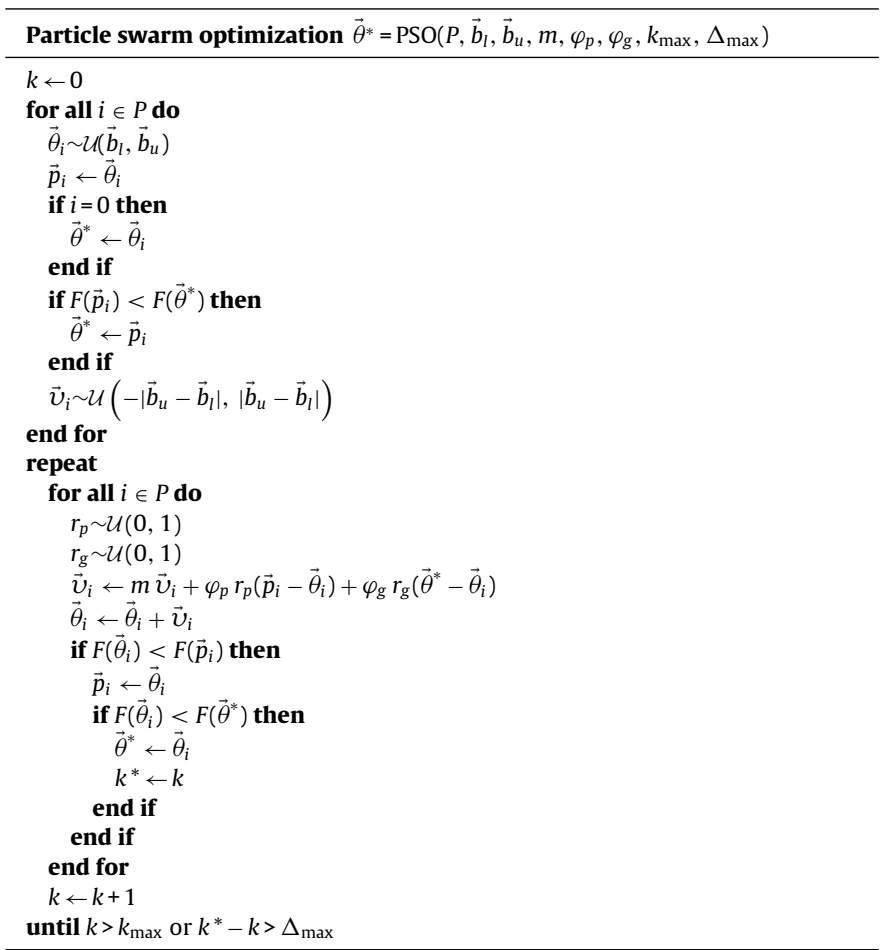

There are several parameters that are of significance for the proper operation of PSO:

- The parameter $P$ denotes the population size of the swarm.

- The parameters $\vec{b}_{l}$ and $\vec{b}_{u}$ represent the lower and upper bounds of the search space. They are constraints of the problem rather than user-set parameters.

- The parameter $m$ denotes the inertia of the particles having an impact on how much particles tend not to change their current velocities at each iteration.

- The social and global cognitive parameters $\varphi_{p}$ and $\varphi_{g}$ determine the amount of the contributions of each particle's own knowledge and the entire swarm population's knowledge, respectively, while updating the particle velocities.

- The parameters $k_{\max }$ and $\Delta_{\max }$, which are the iteration number limit and the maximum solution update rate, respectively, define the stopping condition of the algorithm.

The selection of these parameters has been studied extensively as PSO performance immensely depends on them. It is recommended to set $0.75<m<1$ in order to introduce the real dynamic system feature that bird flocks have, into the flow of the optimization. The correlation between the population size parameter $P$ and the dimension of the unknown parameter vector $\vec{\theta}$ is shown for successful operation. The parameters $\varphi_{p}$ and $\varphi_{g}$ are generally set to a value greater than one. The selection of $k_{\max }$ and $\Delta_{\max }$ is generally based on a trial-and-error approach.

\section{References}

[1] K. Altun, B. Barshan, O. Tunçel, Comparative study on classifying human activities with miniature inertial and magnetic sensors, Pattern Recognit. 43 (October (10)) (2010) 3605-3620.
[2] A. Yurtman, B. Barshan, Automated evaluation of physical therapy exercises using multi-template dynamic time warping on wearable sensor signals, Comput. Methods Programs Biomed. 117 (November (2)) (2014) 189-207.

[3] A.M. Sabatini, Inertial sensing in biomechanics: a survey of computational techniques bridging motion analysis and personal navigation, in: Computational Intelligence for Movement Sciences: Neural Networks and Other Emerging Techniques, Idea Group Publishing, Hershey, PA, USA, 2006, pp. 70-100.

[4] S. Abbate, M. Avvenuti, P. Corsini, A. Vecchio, J. Light, Monitoring of human movements for fall detection and activities recognition in elderly care using wireless sensor network: a survey, in: Application-Centric Design Book, 1st ed., InTech, Rijeka, Croatia, 2010, pp. 147-166 (Chapter 9).

[5] A.T. Özdemir, B. Barshan, Detecting falls with wearable sensors using machine learning techniques, Sensors 14 (June (6)) (2014) 10691-10708.

[6] B. Barshan, H.F. Durrant-Whyte, Inertial navigation systems for mobile robots, IEEE Trans. Robotics Autom. 11 (June (1)) (1995) 328-342.

[7] B. Barshan, H.F. Durrant-Whyte, Evaluation of a solid-state gyroscope for robotics applications, IEEE Trans. Instrum. Meas. 44 (February (1)) (1995) 61-67.

[8] C.-W. Tan, S. Park, Design of accelerometer-based inertial navigation systems, IEEE Trans. Instrum. Meas. 54 (December (6)) (2005) 2520-2530.

[9] P.C. Lin, H. Komsuoglu, D.E. Koditschek, Sensor data fusion for body state estimation in a hexapod robot with dynamical gaits, IEEE Trans. Robot. 22 (October (5)) (2006) 932-943.

[10] D.H. Titterton, J.L. Weston, Strapdown Inertial Navigation Technology, 2nd ed., The American Institute of Aeronautics and Astronautics, Reston, VA, USA, 1979.

[11] B. Barshan, Gyroscopes, Wiley Encyclopedia of Electrical and Electronics Engineering, vol. 8, John Wiley \& Sons, Inc., Hoboken, NJ, USA, 1999, pp. 545-567.

[12] F. Martel, P.K. Pal, M.L. Psiaki, Three-axis attitude determination via Kalman filtering of magnetometer data, in: Flight Mechanics/Estimation Theory Symposium, NASA Goddard Space Flight Center, MD, USA, May 1988, pp. 344-367.

[13] A. Schmidt, Archeology, magnetic methods, in: D. Gubbins, E. Herrero-Bervera (Eds.), Encylopedia of Geomagnetism and Paleomagnetism, Springer, New York, 2007, pp. 23-31.

[14] Y. Yang, Magnetometer and differential carrier phase GPS-aided INS for advanced vehicle control, IEEE Trans. Robotics Autom. 19 (April (2)) (2003) 269-282.

[15] VectorNav Technologies, Embedded Navigation Solutions, Inertial Navigation, 2016 http://www.vectornav.com/support/library/imu-and-ins.

[16] S. Sukkarieh, E.M. Nebot, H.F. Durrant-Whyte, A high integrity IMU/GPS navigation loop for autonomous land vehicle applications, IEEE Trans. Robotics Autom. 15 (June (3)) (1999) 572-578.

[17] H. Zhang, Y. Wu, W. Wu, M. Wu, X. Hu, Improved multi-position calibration for inertial measurement units, Meas. Sci. Technol. 21 (1) (2010) 015107

[18] J.K. Bekkeng, Calibration of a novel MEMS inertial reference unit, IEEE Trans. Instrum. Meas. 58 (June (6)) (2009) 1967-1974

[19] IEEE, IEEE Std. 952-1997 Standard Specification Format Guide and Test Procedure for Single-Axis Interferometric Fiber Optic Gyros, 2008.

[20] P. Minha, Error Analysis and Stochastic Modeling of MEMS Based Inertial Sensors for Land Vehicle Navigation Applications, PhD thesis, Dept. Geomatics Eng., Univ. of Calgary, Alberta, Canada, 2004.

[21] G. Seçer, Deterministic and Stochastic Error Modeling of Inertial Sensors and Magnetometers, MSc thesis, Bilkent Univ., Dept. Electrical and Electronics Eng., Ankara, Turkey, 2012, August http://hdl.handle.net/11693/16681.

[22] 3DM-GX2®|Microstrain, MicroStrain, Williston VT, USA, 2016 http://www. microstrain.com/inertial/3DM-GX2.

[23] MTi and MTx User Manual and Technical Documentation, Xsens Technologies B.V., Enschede, The Netherlands, 2016 http://www.xsens.com.

[24] X. Li, Z. Li, A new calibration method for tri-axial field sensors in strap-down navigation systems, Meas. Sci. Technol. 23 (10) (2012) 105105.

[25] B. Fang, W. Chou, L. Ding, An optimal calibration method for a MEMS inertial measurement unit, Int. J. Adv. Robotic Syst. 11 (14) (2014).

[26] P. Batista, C. Silvestre, P. Oliveira, B. Cardeira, Accelerometer calibration and dynamic bias and gravity estimation: analysis, design, and experimental evaluation, IEEE Trans. Control Syst. Technol. 19 (September (5)) (2011) 1128-1137.

[27] T. Nieminen, J. Kangas, S. Suuriniemi, L. Kettunen, An enhanced multi-position calibration method for consumer-grade inertial measurement units applied and tested, Meas. Sci. Technol. 21 (10) (2010) 105204.

[28] B. Barshan, G. Seçer, Improved deterministic measurement model for consumer-grade accelerometers, Electron. Lett. 52 (April (7)) (2016) $529-531$

[29] J.F. Vasconcelos, G. Elkaim, C. Silvestre, P. Oliveira, B. Cardeira, Geometric approach to strapdown magnetometer calibration in sensor frame, IEEE Trans. Aerosp. Electron. Syst. 47 (April (2)) (2011) 1293-1306.

[30] C. Konvalin, Motion/velocity/displacement compensating for tilt, hard-iron, and soft-iron effects, Sensors Online, 2009, December http://www. sensorsmag.com/sensors/motion-velocity-displacement/compensating-tilthard-iron-and-soft-iron-effects-6475.

[31] C.C. Foster, G.H. Elkaim, Extension of a two-step calibration methodology to include nonorthogonal sensor axes, IEEE Trans. Aerosp. Electron. Syst. 44 (July (3)) (2008) 1070-1078 
[32] J.C. Lötters, J. Schipper, P.H. Veltink, W. Olthuis, P. Bergveld, Procedure for in-use calibration of triaxial accelerometers in medical applications, Sens. Actuators A: Phys. 68 (June (1-3)) (1998) 221-228.

[33] S. Levy, A.E. McPherson, E.V. Hobbs, Calibration of accelerometers, J. Res. Natl. Bur. Stand. 41 (November) (1948) 359-369.

[34] A. Kourepenis, Performance of MEMS inertial sensors, in: IEEE Position Location and Navigation Symposium, Palm Springs, CA, USA, April 1998, pp. $1-8$.

[35] L. Ojeda, H. Chung, J. Borenstein, Precision-calibration of fiber-optics gyroscopes for mobile robot navigation, in: Proc. IEEE Int. Conf. Robotics Autom., vol. 3, San Francisco, CA, USA, 24-28 April 2000, pp. 2064-2069.

[36] M. Tehrani, Ring laser gyro data-analysis with cluster sampling technique, Proc. SPIE 412 (1983) 207-220.

[37] H. Hou, Modeling of Inertial Sensors Using Allan Variance, MSc thesis, Dept. Geomatics Eng., Univ. of Calgary, Alberta, Canada, 2004.

[38] N. El-Sheimy, H. Hou, X. Niu, Analysis and modeling of inertial sensors using Allan variance, IEEE Trans. Instrum. Meas. 57 (January (1)) (2008) 140-149.

[39] W.T. Fong, S.K. Ong, A.Y.C. Nee, Methods for in-field user calibration of an inertial measurement unit without external equipment, Meas. Sci. Technol. 19 (8) (2008) 085202

[40] Y. Li, X. Niu, Q. Zhang, H. Zhang, Y. Shi, An in situ hand calibration method using a pseudo-observation scheme for low-end inertial measurement units, Meas. Sci. Technol. 23 (10) (2012) 105104

[41] E.M. Nebot, H.F. Durrant-Whyte, Initial calibration and alignment of low-cost inertial navigation units for land vehicle applications, J. Robotic Syst. 16 (February (2)) (1999) 81-92.

[42] A. Saxena, G. Gupta, V. Gerasimov, S. Ourselin, In use parameter estimation of inertial sensors by detecting multilevel quasi-static states, in:

Knowledge-Based Intelligent Information and Engineering Systems, Lect. Notes Comput. Sci., in: R. Khosla, R.J. Howlett, J.C. Lakhmi (Eds.), Springer, Berlin, Heidelberg, 2005, pp. 595-601.

[43] B. Li, J. Lu, W. Xiao, T. Lin, In-field fast calibration of FOG-based MWD IMU for horizontal drilling, Meas. Sci. Technol. 26 (3) (2015) 035001

[44] D. Gebre-Egziahber, G.H. Elkaim, J.D. Powell, B.W. Parkinson, Calibration of strapdown magnetometers in magnetic field domain, J. Aerospace Eng. 19 (April (2)) (2006) 87-102.

[45] J. Vcelak, P. Ripka, J. Kubik, A. Platil, P. Kaspar, AMR navigation systems and methods of their calibration, Sens. Actuators A: Phys. 123 (September) (2005) $122-128$.

[46] V. Renauding, M.H. Afzal, G. Lachapelle, Complete triaxis magnetometer calibration in the magnetic domain, J. Sens. (November) (2010) 359-369.

[47] D. Gebre-Egziabher, G.H. Elkaim, J.D. Powell, B.W. Parkinson, A non-linear, two-step estimation algorithm for calibrating solid-state strapdown magnetometers, in: Proc. Eighth Saint Petersburg Int. Conf. on Integrated Navigation Syst., Saint Petersburg, Russia, 28-30 May 2001, pp. 28-37.

[48] Z. Wu, X. Hu, M. Wu, J. Cao, Constrained total least-squares calibration of three-axis magnetometer for vehicular applications, Meas. Sci. Technol. 24 (9) (2013) 095003

[49] J.C. Springmann, J.W. Cutler, Attitude-independent magnetometer calibration with time-varying bias, J. Guid. Control Dyn. 35 (August (4)) (2012) 1080-1088.

[50] J. Metge, R. Mégret, A. Giremus, Y. Berthoumieu, T. Décamps, Calibration of an inertial-magnetic measurement unit without external equipment, in the presence of dynamic magnetic disturbances, Meas. Sci. Technol. 25 (12) (2014) 125106.

[51] ACUTRONIC Switzerland Ltd., Inertial Guidance Test Instrument, 3-Axis Motion Simulator Series AC3357, 2011 http://www.acutronic.com/ch/ products/3-axis/product-detail/view/3-axis-motion-simulator-series-ac3357. html? nar=2\&cHash=c49a557c2b8ee11462027ecccf338bd8, Datasheet: http://www.acutronic.com/uploads/tx_AcutronicProducts/Datasheet-3-AxisRate-Table-AC3357.pdf (Rev B, June 2011).

[52] Z.C. Wu, Z.F. Wang, Y. Ge, Gravity based online calibration for monolithic triaxial accelerometers' gain and offset drift, in: Proc. 4th World Congress on Intelligent Control and Automation, vol. 3, Shanghai, China, 10-14 June 2002, pp. 2171-2175.

[53] J. Borenstein, Experimental evaluation of a fiber optics gyroscope for improving dead reckoning accuracy in mobile robots, in: Proc. IEEE Int. Conf. Robotics Autom. vol. 4, Leuven, Belgium, 16-21 May 1998, pp. 3456-3461.

[54] E. Dorveaux, D. Vissiere, A. Martin, N. Petit, Iterative calibration method for inertial and magnetic sensors, in: Proc. Joint 48th IEEE Conf. Decision and Control and 28th Chinese Control Conf., Shangai, China, 16-18 December 2009, pp. 8296-8303.
[55] S. Bonnet, C. Bassompierre, C. Godin, S. Lesecq, A. Barraud, Calibration methods for inertial and magnetic sensors, Sens. Actuators A: Phys. 156 (December (2)) (2009) 302-311

[56] W. Gander, G.H. Golub, R. Strebel, Least-squares fitting of circles and ellipses, BIT Numer. Math. 34 (December (4)) (1994) 558-578.

[57] W.H. Press, B.P. Flannery, S.A. Teukolsky, W.T. Vetterling, Numerical Recipes in C: The Art of Scientific Computing, 2nd ed., Cambridge University Press, Cambridge, UK, 1992, pp. 681-688.

[58] J. Kennedy, Particle swarm optimization, in: C. Sammut, G.I. Webb (Eds.), Encylopedia of Machine Learning, Springer, New York, 2011, pp. 760-766.

[59] Y. Shi, C. Eberhart, Parameter selection in particle swarm optimization, in: Evolutionary Programming VII, Lect. Notes Comput. Sci., 1447 1998, pp. 591-600.

[60] National Geophysical Data Center, The World Magnetic Model, 2015 http:// www.ngdc.noaa.gov/geomag/WMM/DoDWMM.shtml.

[61] G. Seçer, B. Barshan, Observability Analysis of Proposed Deterministic Measurement Models for Accelerometers and Magnetometers, Tech. Report, ResearchGate, 2016, http://dx.doi.org/10.13140/RG.2.1.2685.8008.

[62] M. Kok, T.B. Schön, Maximum likelihood calibration of magnetometers using inertial sensors, in: Proc. 19th World Congress of the IFAC, Cape Town, South Africa, 24-29 August 2014, pp. 92-97.

[63] M.I.A. Lourakis, A Brief Description of the Levenberg-Marquardt Algorithm Implemented by levmar, Tech. Report, Institute of Computer Science, Foundation for Research and Technology-Hellas (FORTH), Crete, Greece, 2005

[64] A. Ali, S. Siddharth, Z. Syed, N. El-Sheimy, Swarm optimization-based magnetometer calibration for personal handheld devices, Sensors 12 (9) (2012) 12455-12472.

[65] Z. Wu, Y. Wu, X. Hu, M. Wu, Calibration of three-axis magnetometer using stretching particle swarm optimization algorithm, IEEE Trans. Instrum. Meas. 62 (February (2)) (2013) 281-292.

[66] Q. Cai, N. Song, G. Yang, Y. Liu, Accelerometer calibration with nonlinear scale factor based on multi-position observation, Meas. Sci. Technol. 24 (10) (2013) 105002

[67] Wikipedia, Geodetic System-Wikipedia, The Free Encyclopedia, 2016 http:// en.wikipedia.org/wiki/Geodetic_system.

\section{Biographies}

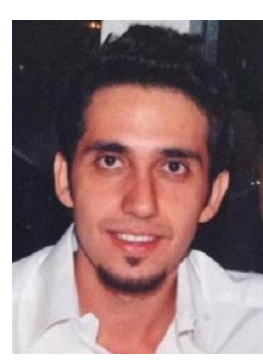

Gorkem Secer received a B.S. degree from Istanbul Technical University in 2009 and an M.S. degree from Bilkent University, Ankara, Turkey in 2012 both in electrical and electronics engineering. Currently, he is a Ph.D. candidate at Middle East Technical University, Department of Computer Engineering, Ankara, Turkey, and works as a system engineer at Roketsan Inc., Ankara, Turkey. His research interest are in instrumentation, robotics, and control systems.

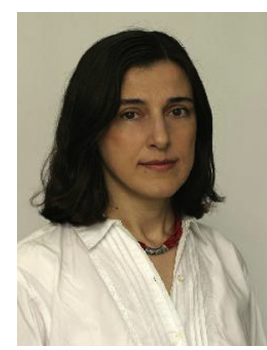

Billur Barshan received B.S. degrees in both electrical engineering and physics from Boğaziçi University in Istanbul, Turkey in 1986 and her M.S. and Ph.D. degrees in electrical engineering from Yale University in the U.S.A. in 1988 and 1991, respectively. Dr. Barshan was a research assistant at Yale from 1987 to 1991 and a postdoctoral researcher at the Robotics Research Group at the University of Oxford in the U.K. from 1991 to 1993. In 1993, she joined the faculty of Bilkent University in Ankara, Turkey, where she is a professor in the Department of Electrical and Electronics Engineering. Dr. Barshan is also the founder of the Robotics and Sensing Laboratory in the same department. She is the recipient of the 1994 Nakamura Prize for the most outstanding paper at the 1993 IEEE/RSJ Intelligent Robots and Systems International Conference, the 1998 TÜBITAK Young Investigator Award and the 1999 Mustafa N. Parlar Foundation Research Award. Dr. Barshan's current research interests include instrumentation and measurement, intelligent sensing, sensor-based robotics, ultrasonic, optical, and inertial sensing, multi-sensor data fusion, and wearable sensing. 